\title{
Equilibrative nucleoside transporter 1 (ENT1) regulates postischemic blood flow during acute kidney injury in mice
}

\author{
Almut Grenz,1 Jessica D. Bauerle, ${ }^{1}$ Julee H. Dalton, ${ }^{1}$ Douglas Ridyard, ${ }^{1}$ \\ Alexander Badulak, ${ }^{1}$ Eunyoung Tak, ${ }^{1}$ Eóin N. McNamee, ${ }^{1}$ Eric Clambey, ${ }^{1}$ Radu Moldovan, ${ }^{2}$ \\ German Reyes, ${ }^{3}$ Jost Klawitter, ${ }^{1}$ Kelly Ambler, ${ }^{4}$ Kristann Magee, ${ }^{1}$ Uwe Christians, ${ }^{1}$ \\ Kelley S. Brodsky, ${ }^{1}$ Katya Ravid, ${ }^{5}$ Doo-Sup Choi, ${ }^{6}$ Jiaming Wen, ${ }^{7}$ Dmitriy Lukashev, ${ }^{8}$ \\ Michael R. Blackburn, ${ }^{7}$ Hartmut Osswald, ${ }^{9}$ Imogen R. Coe,$^{3}$ Bernd Nürnberg, ${ }^{9}$ \\ Volker H. Haase, ${ }^{10}$ Yang Xia, ${ }^{7}$ Michail Sitkovsky, ${ }^{8}$ and Holger K. Eltzschig ${ }^{1}$
}

\begin{abstract}
${ }^{1}$ Mucosal Inflammation Program, Department of Anesthesiology and ${ }^{2}$ Advanced Light Microscopy Core Facility, School of Medicine, University of Colorado Denver, Anschutz Medical Campus, Aurora, Colorado, USA. ${ }^{3}$ Department of Biology, York University, Toronto, Ontario, Canada. ${ }^{4}$ Department of Cardiology, University of Colorado Denver, Anschutz Medical Campus, Aurora, Colorado, USA. ${ }^{5}$ Department of Biochemistry, Boston University School of Medicine, Boston, Massachusetts, USA. ${ }^{6}$ Department of Molecular Pharmacology and Experimental Therapeutics Mayo Clinic College of Medicine, Rochester, Minnesota, USA. 'Department of Biochemistry and Molecular Biology, The University of Texas Health Science Center at Houston, Houston, Texas, USA. ${ }^{8} \mathrm{New}$ England Inflammation and Tissue Protection Institute, Northeastern University, Boston, Massachusetts, USA. ${ }^{9}$ Department of Pharmacology and Experimental Therapy, University of Tübingen, Tübingen, Germany. ${ }^{10}$ Departments of Medicine, Cancer Biology, and Molecular Physiology and Biophysics, Vanderbilt University School of Medicine, Nashville, Tennessee, USA.
\end{abstract}

A complex biologic network regulates kidney perfusion under physiologic conditions. This system is profoundly perturbed following renal ischemia, a leading cause of acute kidney injury (AKI) - a life-threatening condition that frequently complicates the care of hospitalized patients. Therapeutic approaches to prevent and treat AKI are extremely limited. Better understanding of the molecular pathways promoting postischemic reflow could provide new candidate targets for AKI therapeutics. Due to its role in adapting tissues to hypoxia, we hypothesized that extracellular adenosine has a regulatory function in the postischemic control of renal perfusion. Consistent with the notion that equilibrative nucleoside transporters (ENTs) terminate adenosine signaling, we observed that pharmacologic ENT inhibition in mice elevated renal adenosine levels and dampened AKI. Deletion of the ENTs resulted in selective protection in Ent1 $1^{-/}$mice. Comprehensive examination of adenosine receptor-knockout mice exposed to AKI demonstrated that renal protection by ENT inhibitors involves the A2B adenosine receptor. Indeed, crosstalk between renal Ent1 and Adora2b expressed on vascular endothelia effectively prevented a postischemic no-reflow phenomenon. These studies identify ENT1 and adenosine receptors as key to the process of reestablishing renal perfusion following ischemic AKI. If translatable from mice to humans, these data have important therapeutic implications.

\section{Introduction}

Acute kidney injury (AKI) is clinically defined by an abrupt reduction in kidney function (e.g., a decrease in glomerular filtration rate $[\mathrm{GFR}]$ ), occurring over a period of minutes to days. AKI is frequently caused by an obstruction of renal blood flow (renal ischemia) and represents an important cause of morbidity and mortality of patients (1-3). Indeed, a recent study revealed that only a mild increase $(0.3 \mathrm{mg} / \mathrm{dl})$ in the serum creatinine level is associated with a $70 \%$ greater risk of death than in patients without this increase $(2,3)$. Particularly for surgical patients, AKI represents a significant threat. For example, surgical procedures requiring cross-clamping of the aorta and renal vessels are associated with a rate of AKI of up to $30 \%$ (4). Similarly, AKI after cardiac surgery occurs in up to $10 \%$ of patients under normal circumstances and is associated with dramatic increases in mortality (5). In addition, patients with sepsis frequently go on to develop AKI, and the combination of moder-

Authorship note: Almut Grenz, Jessica D. Bauerle, and Julee H. Dalton contributed equally to this work.

Conflict of interest: The authors have declared that no conflict of interest exists. Citation for this article: J Clin Invest. 2012;122(2):693-710. doi:10.1172/JCI60214. ate sepsis and AKI is associated with a $70 \%$ rate of mortality. Unfortunately, therapeutic approaches to prevent or treat AKI are extremely limited, as the majority of interventional trials in AKI have failed in humans $(6,7)$. Therefore, the search for novel therapeutic modalities to prevent or treat AKI presently represents an area of intense investigation (8).

Previous studies had implicated the signaling molecule adenosine in tissue adaptation to hypoxia $(9,10)$. Indeed, extracellular adenosine production from precursor molecules (nucleotides such as ATP, ADP, or AMP) (11) is dramatically increased during conditions of limited oxygen availability (12). Adenosine signals through four distinct adenosine receptors (ARs) (ADORA1, ADORA2A, ADORA2B, ADORA3) $(13,14)$, and studies show that hypoxia also enhances adenosine signaling by transcriptional increases in AR levels (12). While the mechanisms remain poorly understood, several studies have shown that adenosine generation and signaling are protective in models of ischemia and reperfusion of the heart $(15,16)$, the liver $(17)$, or the intestine $(18,19)$.

During conditions of limited oxygen availability, the adenosine gradient across the cell membrane is directed from the extracellular toward the intracellular compartment. In this regard, equilibrative nucleoside transporters (ENTs) represent a 


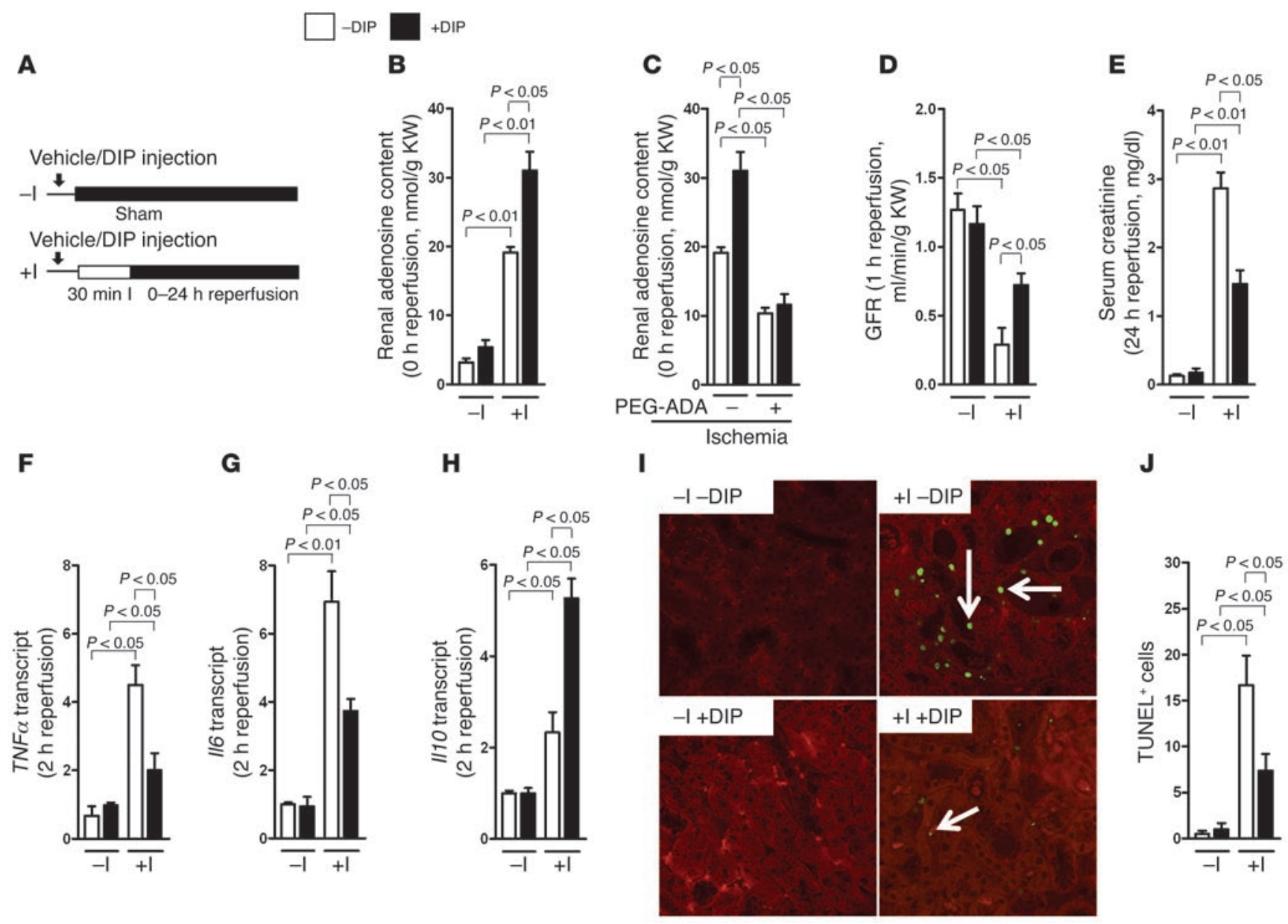

Figure 1

Renal function and inflammation following inhibition of adenosine transporters during renal ischemia. (A) Experimental setup to study renal ischemia and reperfusion; DIP, dipyridamole $(0.25 \mathrm{mg} / 25 \mathrm{~g}$ mouse i.v., 1 hour before renal ischemia); $-\mathrm{I}$, sham-operated controls; $+\mathrm{I}, 30 \mathrm{minutes}$ of isolated renal artery occlusion and reperfusion time as indicated. (B) Renal adenosine content measured immediately following 30 minutes of renal ischemia in mice treated with the inhibitor of ENTs dipyridamole or vehicle (+DIP; -DIP; $n=4-6)$. KW, kidney weight. (C) Renal adenosine content in mice pretreated with PEG-ADA (5 U/mouse 24 hours before renal ischemia; $n=4)$. (D) GFR following 30 minutes of renal ischemia and 1 hour of reperfusion $(n=4-6)$. (E) Serum creatinine levels following 30 minutes of renal ischemia and 24 hours of reperfusion $(n=4-6)$. $(\mathbf{F}-\mathbf{H}) T N F \alpha$, I/6, and $/ / 10$ transcript levels after 30 minutes of renal ischemia and 2 hours of reperfusion by real-time RT-PCR relative to the housekeeping gene $\beta$-actin $(n=4-6)$. (I) Histologic staining for apoptosis (as determined by TUNEL staining) in kidneys exposed to 30 minutes of ischemia and 24 hours of reperfusion (original magnification, $\times 400$; fluorescent green [arrows]: staining for apoptosis; 1 representative image of 3 is shown). (J) Quantification of renal apoptosis by counting positive cells in $5-10 \times 400$ fields.

critical bottleneck in terminating extracellular adenosine signaling events (20). These transporters allow for passive flow of adenosine across the cell membrane along its gradient (21). Indeed, pharmacologic ENT inhibition represents a means of enhancing extracellular adenosine signaling during hypoxia $(22,23)$. In the present study, we sought to determine whether ENTs play a functional role during ischemic AKI. Surprisingly, our investigations revealed a crosstalk pathway between renal ENT1 and vascular ARs in controlling postischemic reflow of the kidneys (24), thereby protecting the kidneys from ischemic AKI.

\section{Results}

ENT inbibition is associated with elevated adenosine and kidney protection from AKI. Limited oxygen availability is known to enhance extracellular adenosine concentrations (25-28), thus resulting in a transcellular adenosine gradient directed from the extracellular toward the intracellular cell compartment. This gradient promotes the passive flow of adenosine along its gradient via adenosine transport proteins of the ENT1 or ENT2 type $(21,29)$. Therefore, we hypothesized that pharmacologic inhibition of adenosine transporters represents a means of enhancing extracellular adenosine signaling during ischemic AKI. To address this hypothesis, we pretreated mice with the nonspecific ENT inhibitor dipyridamole and subsequently exposed them to ischemic AKI, utilizing a model of isolated renal artery occlusion (Figure 1A and ref. 30). Consistent with our hypothesis, we observed that renal adenosine levels were elevated following 30 minutes of renal ischemia, with an additional rise in adenosine in mice pretreated with dipyridamole (Figure 1B and Supplemental Figure 1, A and B; supplemental material available online with this article; doi:10.1172/ JCI60214DS1). Moreover, the dipyridamole-elicited elevations in renal adenosine levels were abolished in animals that were 

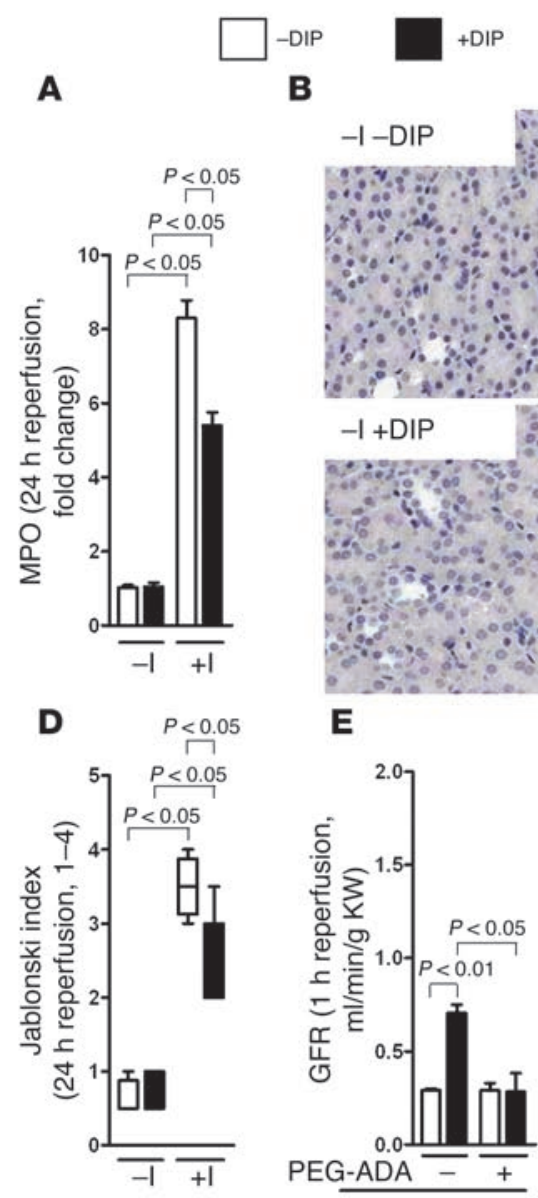

B

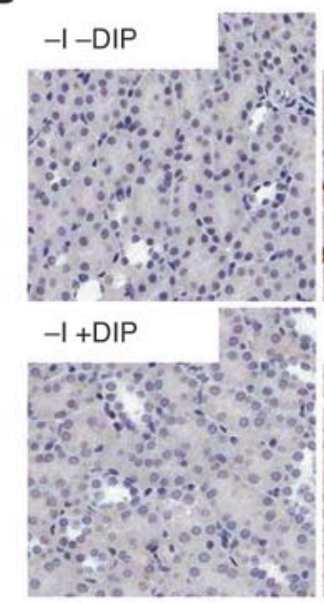

E

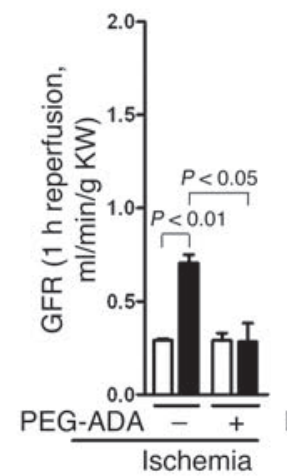

\begin{abstract}
F
\end{abstract}

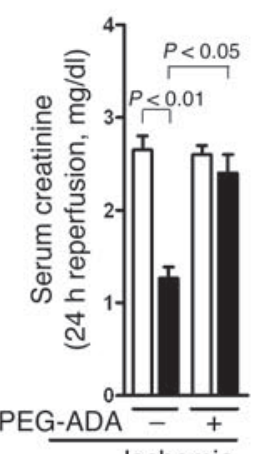

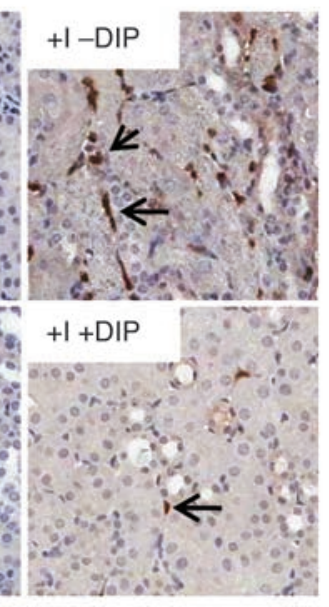

G

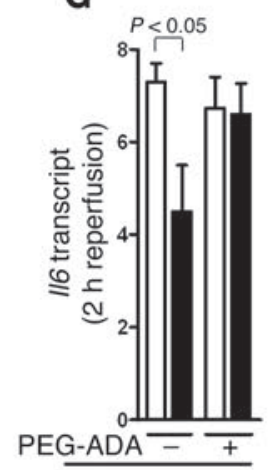

Ischemia
C

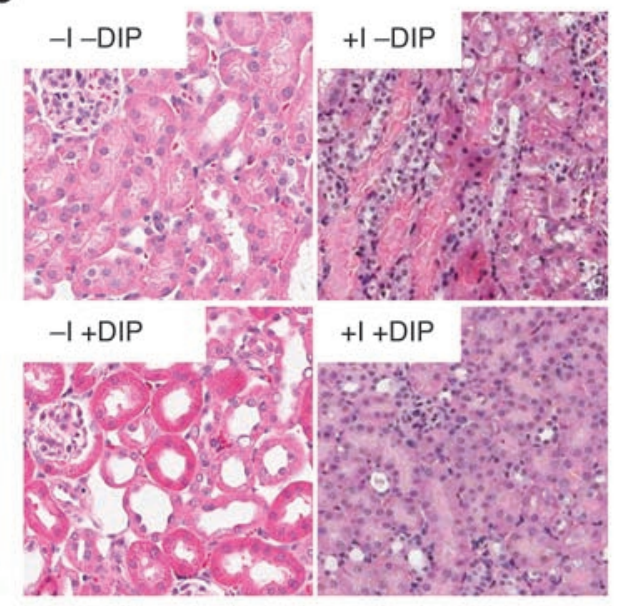

H

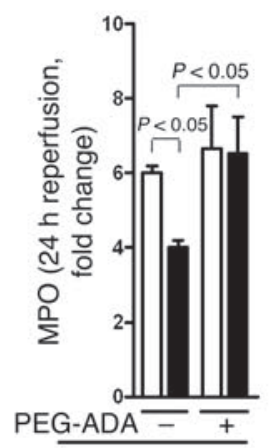

Ischemia
I

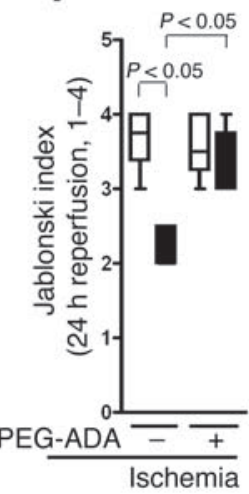

Figure 2

Renal histology following ischemic AKI following treatment with ENT inhibitor dipyridamole, and effect of PEG-ADA during ischemic AKI. (A) Levels of the neutrophil marker MPO in kidneys exposed to 30 minutes of ischemia and 24 hours of reperfusion $(n=4-6)$. (B) Histologic staining for neutrophils (arrows) in kidneys exposed to 30 minutes of ischemia and 24 hours of reperfusion (original magnification, $\times 400 ; 1$ representative image of 4 is shown). (C) Renal histology following 30 minutes of renal ischemia and 24 hours reperfusion (original magnification, $\times 400 ; 1$ representative image of 4 is shown). (D) Mice were pretreated with PEG-ADA (5 U/mouse 24 hours before renal ischemia; $n=3-6)$ and subsequently underwent ischemic AKI in the presence or absence of dipyridamole. Quantification of renal tissue injury according to the Jablonski index $(n=4)$. (E-I) GFR, serum creatinine, I/6, MPO, and histologic assessment of tissue injury by the Jablonski index in mice pretreated with PEG-ADA and subsequently exposed to 30 minutes of renal ischemia and the indicated reperfusion periods $(n=3-6)$.

treated with polyethylene glycol-modified adenosine deaminase (PEG-ADA). As PEG-ADA cannot cross the cell membrane, these findings demonstrate the extracellular nature of the observed rise in adenosine levels (Figure 1C).

Additional studies demonstrated that pretreatment with dipyridamole was associated with improved kidney function in isolated unilateral renal artery occlusion, as well as in a bilateral pedicle occlusion model (Figure 1, D and E, and Supplemental Figure 2, A and B). In addition, dipyridamole treatment attenuated kidney inflammation (Figure 1, F-H). Furthermore TUNEL-positive cells were increased in kidney sections from mice following ischemia compared with controls without ischemia (Figure 1, I and J). Treatment with dipyridamole before renal ischemia reduced TUNEL-positive cells.

Assessment of renal infiltration with neutrophils and histology confirmed kidney protection from ischemic AKI with dipyridamole treatment (Figure 2, A-D). The observed kidney protection with dipyridamole was completely abolished in the presence of
PEG-ADA (Figure 2, E-I), thereby implicating extracellular adenosine in mediating the kidney protection observed with dipyridamole treatment. Together, these findings demonstrate that inhibition of renal adenosine uptake with dipyridamole is associated with elevation of extracellular adenosine levels, concomitant with kidney protection from ischemic injury.

Renal ENTs are repressed during ischemic AKI. Prompted by these findings, we next examined changes in expression of renal ENTs in response to ischemic AKI. Previous studies had indicated that extracellular adenosine uptake during hypoxia predominantly involves ENT1 or ENT2 $(22,23)$. We observed that renal Ent1 and Ent2 expression was repressed during renal ischemia, with higher expressional levels of Ent1 compared with Ent2 (Figure 3, A-C). Subsequent analysis of isolated tubules or glomeruli revealed that Ent1 was predominantly expressed in the tubules (Figure 3D). Based on these findings, we utilized cultured human tubular epithelia (HK-2) cells as an in vitro model for renal adenosine trans- 


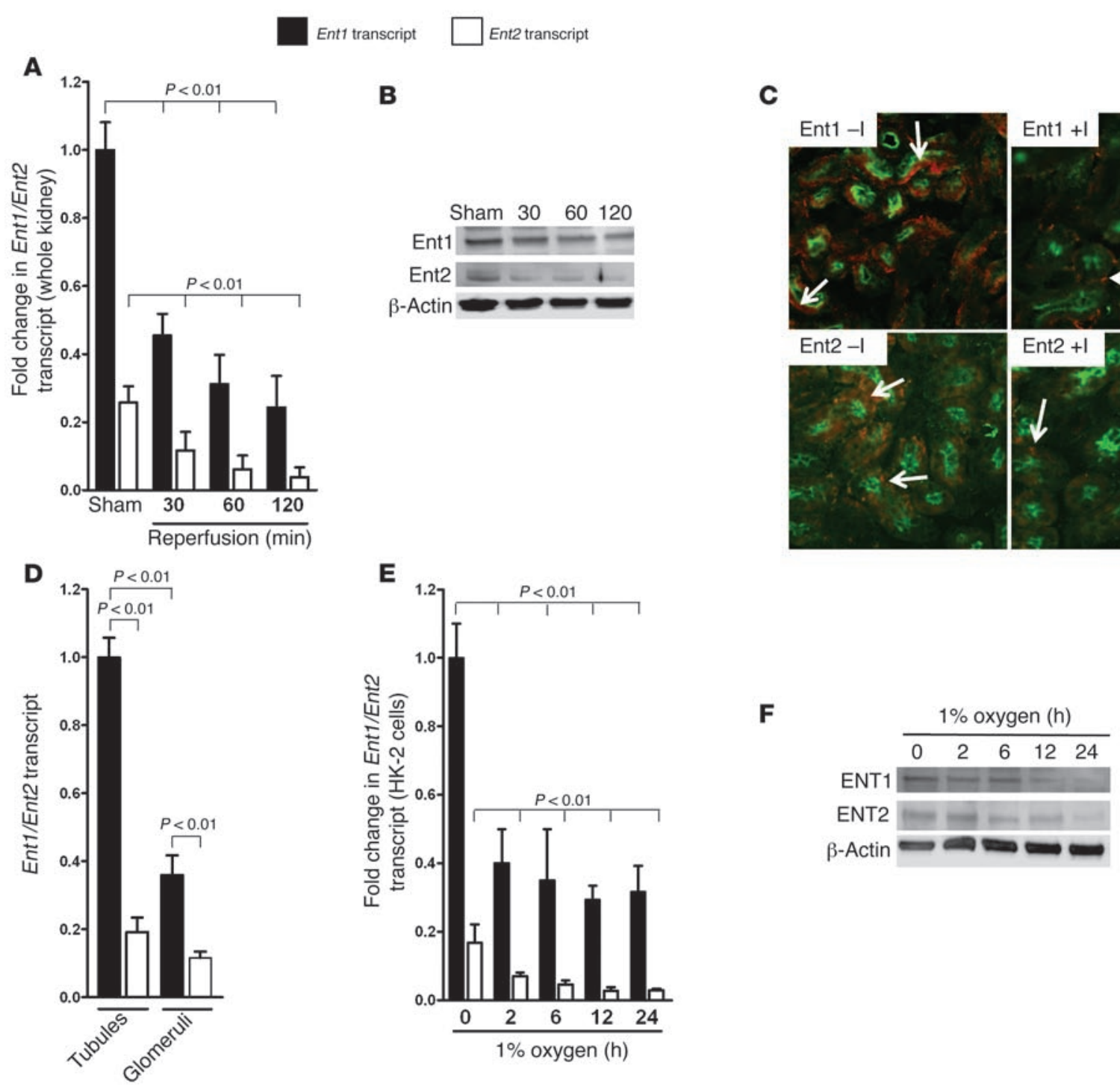

Figure 3

Influence of renal ischemia on ENT expression. (A) Ent1 and Ent2 transcript levels in kidneys exposed to sham operation (Sham) or 30 minutes of ischemia, followed by reperfusion for the indicated time periods assessed by real-time RT-PCR relative to $\beta$-actin ( $n=4$ independent experiments). (B) Ent1 and Ent2 protein levels assessed by Western blotting ( $\beta$-actin to control for loading conditions; 1 representative blot of 3 is shown). (C) Comparison of immunoreactivity for Ent1 and Ent2 in kidneys exposed to 30 minutes of renal ischemia and 2 hours of reperfusion or sham-operated controls (original magnification, $\times 400$; fluorescent green: counterstaining for proximal tubules; fluorescent red [arrows]: staining for Ent1 or Ent2; 1 representative image of 3 is displayed). (D) Relative expression levels of Ent1 or Ent2 transcript in isolated tubules or glomeruli, relative to $\beta$-actin by real-time RT-PCR ( $n=4$ independent experiments). (E) ENT1 and ENT2 transcript levels in cultured HK-2 cells exposed for the indicated time periods to ambient hypoxia ( $1 \%$ oxygen) assessed by real-time RT-PCR relative to $\beta$-actin $(n=4$ independent experiments). (F) ENT1 and ENT2 protein levels from HK-2 cells exposed to indicated time periods to ambient hypoxia (1\% oxygen) and assessed by Western blot ( $\beta$-actin to control for loading conditions; 1 representative blot of 3 is shown).

porter expression and function. Indeed, hypoxia exposure of HK-2 cells was associated with repression of ENT1 or ENT2 transcript and protein levels (Figure 3, E and F). Additional studies with radioactively labeled adenosine that was added to the basolateral or apical compartment of HK-2 cells (Figure 4A) demonstrated that ENT-dependent adenosine transport occurs mainly from the basolateral surface and is repressed following hypoxia exposure (Figure 4, B and C). Moreover, differential biotinylation of individual ENTs revealed that ENT1 and ENT2 were predominantly localized to the basolateral surface (Figure 4, D and E). Together, these studies demonstrate that ischemic AKI is associated with transcriptional repression of ENT1 and ENT2, in conjunction with attenuated basolateral adenosine transport. These findings indicate the likelihood that ENT repression represents an endogenous pathway to protect the kidneys from ischemia via repression of adenosine transporter expression and concomitant elevations in extracellular adenosine concentrations in the tissues.

Selective role for renal ENT1 in kidney protection from ischemia. To address the individual contributions of ENT1 or ENT2 during ischemic AKI, we next performed studies in mice with knockout of 
A

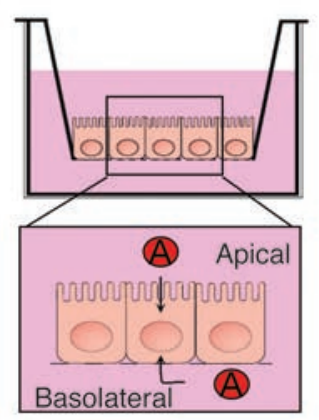

B

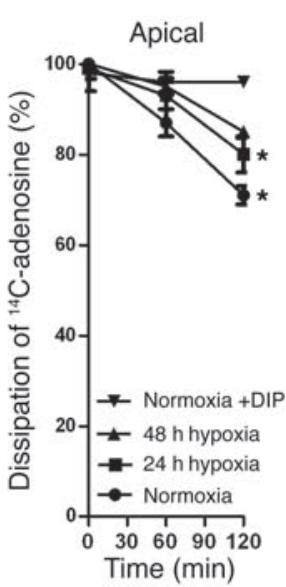

C

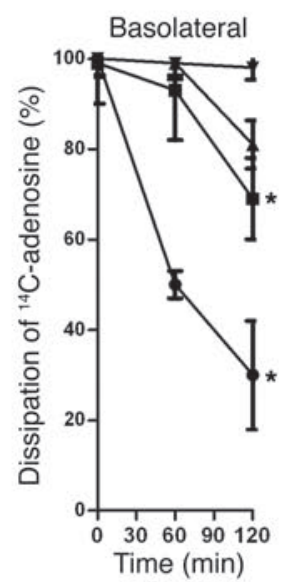

D

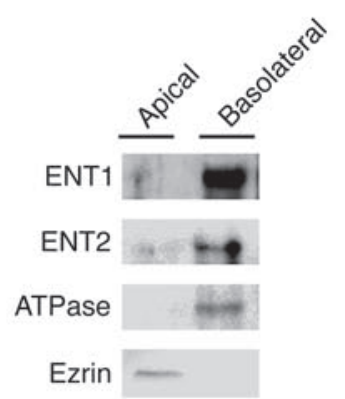

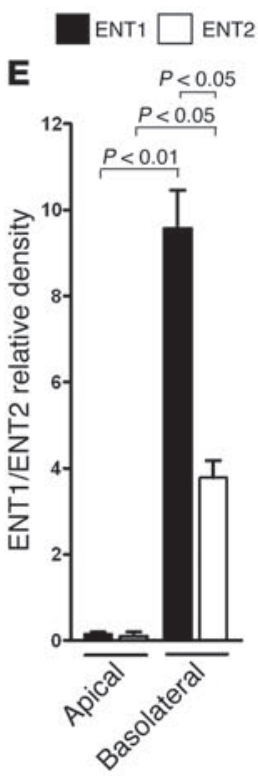

\section{Figure 4}

Influence of renal ischemia on ENT function and cellular localization of ENTs. (A) Model for measuring epithelial adenosine uptake. HK-2 cells were grown on inserts until they were fully confluent (approximately 7 days). Exogenous adenosine ( $5 \mathrm{M}$ adenosine containing $10 \mathrm{nCi}$ of $8-{ }^{14} \mathrm{C}$-adenosine) was added to the basolateral or apical compartment, and dissipation of labeled adenosine was measured at indicated time points. (B) HK-2 cells were maintained under normoxic conditions or exposed to 24 or 48 hours of ambient hypoxia (1\% oxygen). In subsets of experiments, dipyridamole $(10 \mu \mathrm{M})$ was added to the supernatant. Adenosine measurements were performed in triplicate, and 1 representative experiment of 3 is shown. ${ }^{*} P<0.05$ by $t$ test compared with samples treated with $10 \mu \mathrm{M}$ dipyridamole (Normoxia + DIP). (C) Basolateral adenosine dissipation. ${ }^{*} P<0.05$ by $t$ test compared with samples treated with $10 \mu \mathrm{M}$ dipyridamole (Normoxia + DIP). (D) Either apical or basolateral surface proteins of confluent HK-2 cells grown on inserts were biotinylated. Immunoprecipitates were resolved by SDS-PAGE, and resultant Western blots were probed with streptavidin-HRP. Apical (ezrin) or basolateral (ATPase) surface markers were included as controls. A representative experiment of 2 is shown. (E) Quantification by densitometry $(n=2)$.

Ent1 or Ent2 (Supplemental Figure 3, A and B, and Supplemental Figure 4, A and B). As a first step in these studies, we examined the adenosine uptake capacity of isolated tubular epithelia from Ent1 $1^{-/}$ or Ent2 $2^{-/-}$mice. While we observed similar kinetics of adenosine uptake of tubular epithelia isolated from wild-type or Ent2 $2^{-/}$mice (Supplemental Figure 5), adenosine uptake was dramatically attenuated in tubules isolated from Ent1 $1^{-/}$mice (Figure 5A). Consistent with this result, renal adenosine levels following ischemic AKI were similar in wild-type and Ent2-/ mice (Supplemental Figure 6), while there was a dramatic elevation of renal adenosine levels in ischemic kidneys from Ent1 $1^{-/}$mice (Figure 5B). Moreover, Ent1 $1^{-/-}$mice showed protection from ischemic AKI, including improved renal function (Figure 5, C and D, and Supplemental Figure 7, A and B), attenuated kidney inflammation (Figure 5, E-G), attenuated histologic tissue injury (Figure 3, $\mathrm{H}$ and I), and reduced TUNEL-positive cell numbers (Figure 5, J and K). Kidney function was improved in the isolated unilateral renal artery occlusion model as well as in the bilateral pedicle occlusion model (Supplemental Figure 7, A and B). In contrast, Ent2 $2^{-/-}$mice exhibited no signs of increased ischemia tolerance compared with controls (Supplemental Figure 8, A-H). To address the question as to whether the tissue protection observed with Ent1 deletion involves hematopoietic or radiation-resistant cells, we generated Ent1 bone marrow chimeric mice (Supplemental Figure 9). These studies demonstrated that deletion of Ent1 in radiation-resistant tissues mediated the observed tissue protection (Figure 6, A-D). As proof of principle, we also performed kidney-specific reconstitution of human ENT1 into the kidneys of
Ent1 $1^{-/}$mice utilizing a lentiviral transduction system (31). Lentiviral transduction was associated with high levels of human ENT1 following transduction (Figure 6E). Moreover, kidney-specific lentiviral transduction of ENT1 into the kidneys of $\mathrm{Ent1}^{-/-}$mice was associated with abolished kidney protection from ischemic AKI in contrast to Ent1-/- mice transduced with an empty control virus (Figure 6, F-I). Together these studies reveal a selective role for renal ENT1 in regulating kidney protection from ischemia.

ENT-dependent kidney protection from ischemia involves AR signaling. After having shown that pharmacologic inhibition or genetic deletion of ENTs is associated with elevated renal adenosine levels, and concurrent protection from ischemic AKI, we next pursued the hypothesis that Ent-dependent kidney protection involves adenosine signaling. To address this hypothesis, we exposed AR-knockout mice to ischemic AKI and examined the protective effects of dipyridamole. While mice with knockout of Adora1, Adora2a, or Adora3 showed a similar degree of kidney protection mediated by pretreatment with dipyridamole, Adora $2 b^{-/}$mice were not protected by dipyridamole treatment (Figure 7, A-O, and Figure 8, A-E). Moreover, pretreatment with the Adora2b-specific antagonist PSB1115 blocked kidney protection mediated by dipyridamole treatment (Figure 8, F-J). Together, these studies indicate that kidney protection mediated by the ENT inhibitor dipyridamole involves signaling events through Adora2b during ischemic AKI.

Selective deletion of endothelial Adora2 $b$ abolishes ENT-mediated kidney protection. After having demonstrated that kidney protection from ischemic AKI mediated by pharmacologic ENT inhibitors involves 


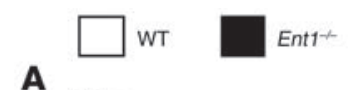

A

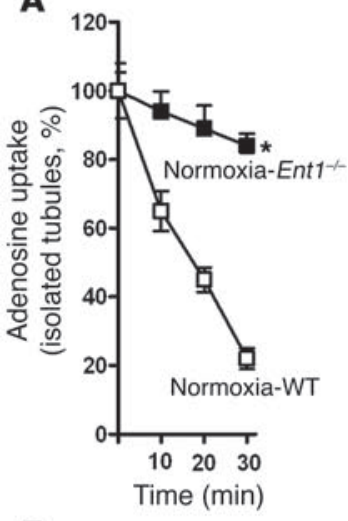

F

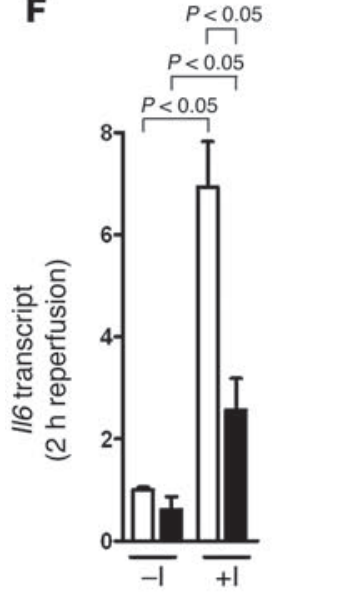

J
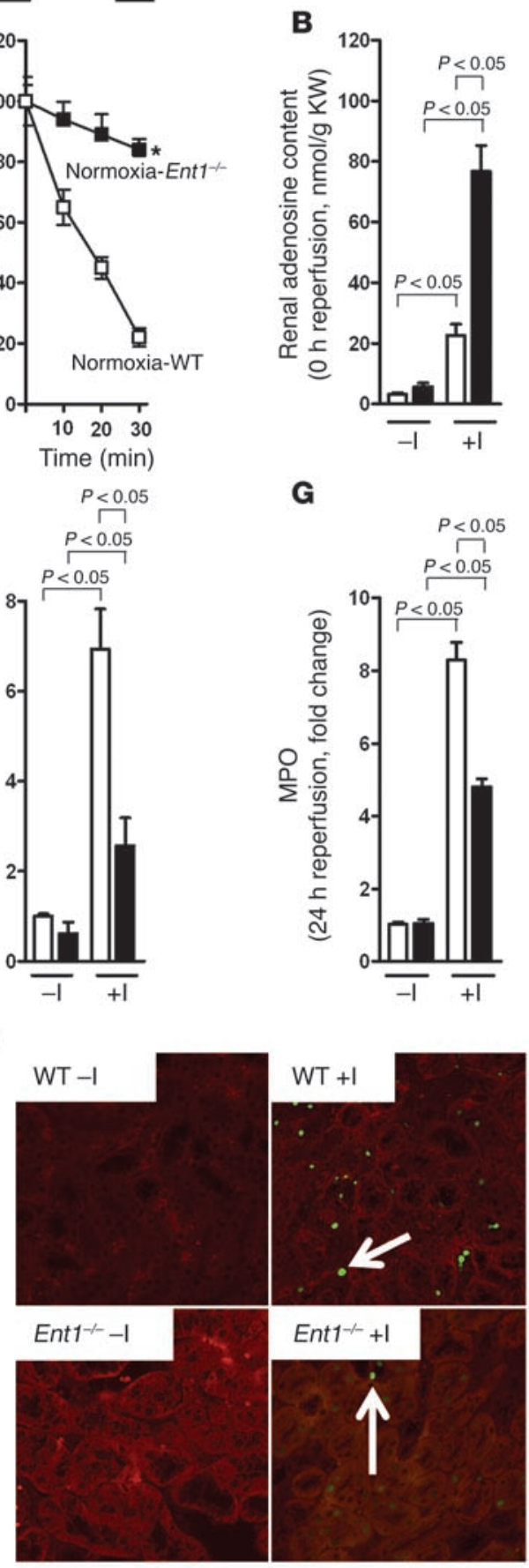

G

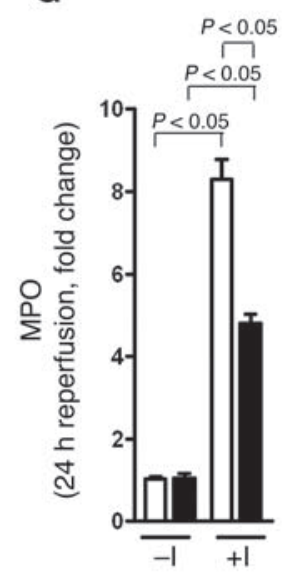

H

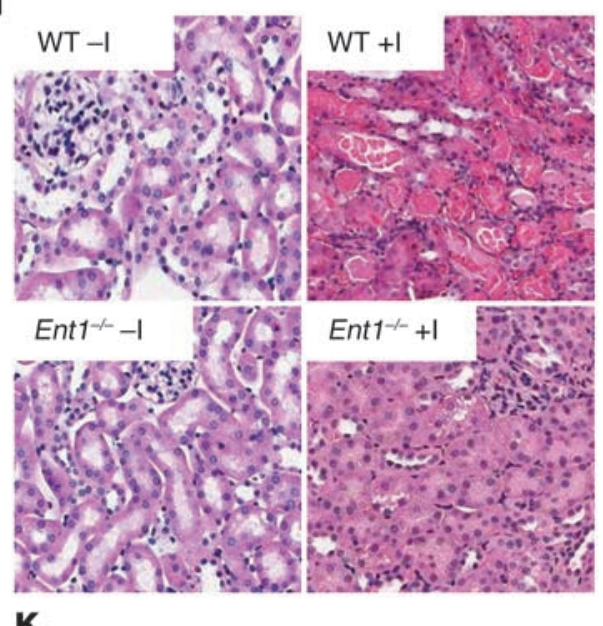

K

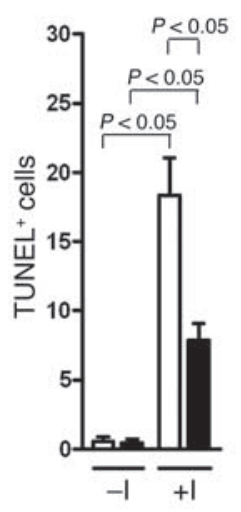

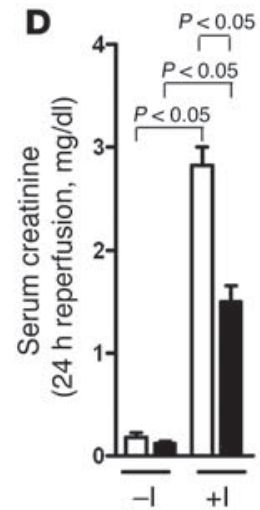

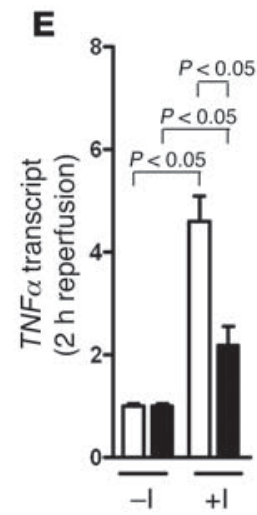

I

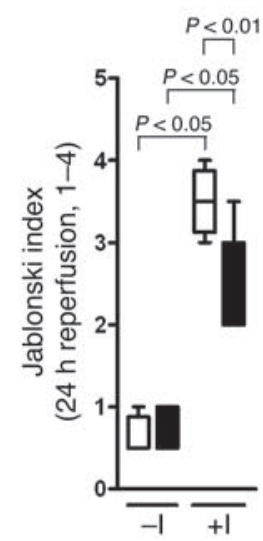

\section{Figure 5}

Characterization of Ent1-/- mice during renal ischemia. (A) Tubules were isolated from wild-type controls or Ent1 ${ }^{-/-}$mice; exogenous adenosine ( $5 \mu \mathrm{M}$ adenosine) was added to the culture medium, and dissipation of labeled adenosine was measured at the indicated time points by HPLC. Measurements were performed in triplicate; 1 representative experiment of 3 is shown. (B) Renal adenosine content measured immediately following 30 minutes of renal ischemia $(n=4-6)$. (C) GFR following 30 minutes of renal ischemia and 1 hour of reperfusion ( $n=4-6)$. (D) Serum creatinine levels following 30 minutes of renal ischemia and 24 hours of reperfusion $(n=4-6)$. (E) TNF $\alpha$ transcript following 30 minutes of renal ischemia and 2 hours of reperfusion by real-time RT-PCR relative to $\beta$-actin $(n=4-6)$. (F) $/ 16$ transcript following 30 minutes of renal ischemia and 2 hours of reperfusion by real-time RT-PCR relative to $\beta$-actin $(n=4)$. (G) Measurement of the neutrophil marker MPO in kidneys exposed to 30 minutes of ischemia and 24 hours of reperfusion $(n=4-6)$. (H) Renal histology following 30 minutes of renal ischemia and 24 hours of reperfusion (original magnification, $\times 400$; 1 representative image of 4 is shown). (I) Quantification of histologic tissue injury utilizing the Jablonski index $(n=4)$. (J) Staining for apoptosis (as determined by TUNEL staining) in kidneys exposed to 30 minutes of ischemia and 24 hours of reperfusion (original magnification, $\times 400$; fluorescent green [arrows]: staining for apoptosis; 1 representative image of 3 is shown). (K) Quantification of renal apoptosis by counting positive cells in $5-10 \times 400$ fields. 

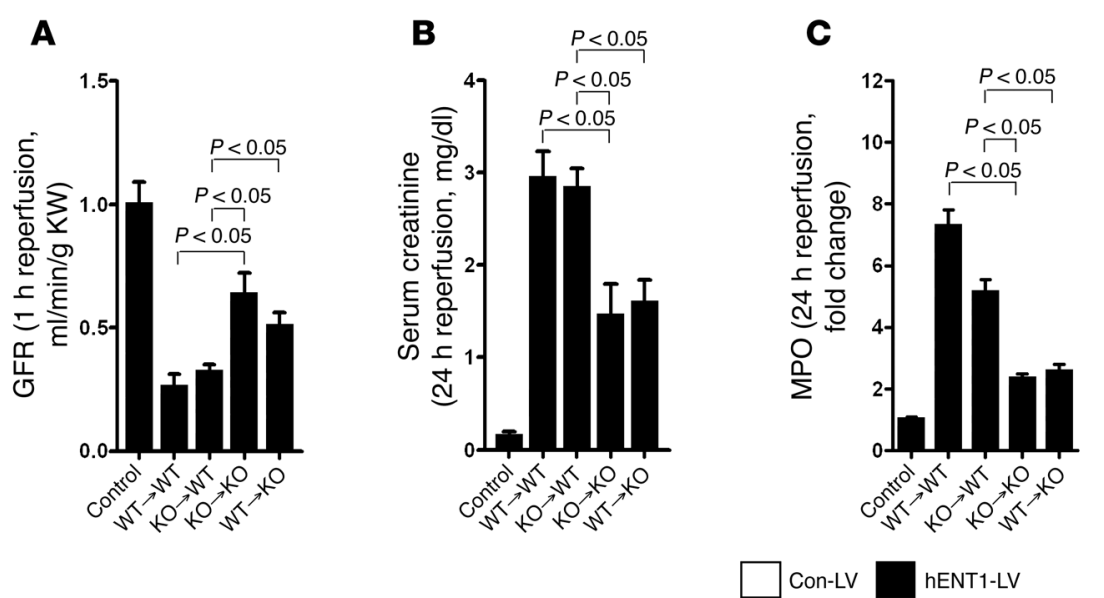

$\mathbf{E}$

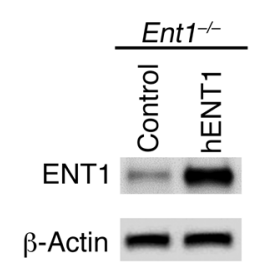

Figure 6

Studies of ischemic AKI in Ent1 bone marrow chimeric mice or in Ent1-1- mice reconstituted with human ENT1. (A-D) Assessment of GFR, serum creatinine, MPO, and quantification of histologic tissue injury in Ent1 bone marrow chimeric mice (Control: sham-operated wild-type mice transplanted with wild-type bone marrow; KO: Ent1-l- mice; $n=4)$. (E) Ent1 ${ }^{-1-}$ kidneys were injected with saline or with a human ENT1-encoding lentivirus. After 24 hours, transcript levels of Ent1 were determined by real-time RT-PCR relative to $\beta$-actin $(n=4)$. $(\mathbf{F}-\mathbf{I})$ Assessment of renal adenosine, GFR, serum creatinine, and MPO tissue levels following 30 minutes of ischemia and indicated reperfusion times $(n=4)$.

Adora2b signaling, we next performed studies to identify the role of Adora2b expressed on different cell types. As previous studies had implicated Adora2b signaling on endothelia (32) or epithelia (33) in organ inflammation, we used a transgenic mouse line with a floxed Adora $2 b$ gene (34) to generate mouse lines with deletion of $A d o r a 2 b$ in vascular endothelia (Supplemental Figure 10, A and B) or tubular epithelia of the kidneys (Supplemental Figure 10, A and B). Indeed, mice with genetic deletion of the Adora2b in tubular epithelia retained dipyridamole-mediated kidney protection from ischemia, indicating that ENT inhibition-mediated kidney protection is independent of Adora2b expression on tubular epithelia (Figure 9, A-E). In contrast, tissue-specific deletion of Adora2b in vascular endothelia completely abolished dipyridamole-dependent kidney protection from ischemic AKI (Figure 9, F-J). Consistent with these findings, studies in an Adora2b reporter model (35) indicated that Adora2b was selectively induced in the renal vasculature following exposure to kidney ischemia (Figure 10A).

Based on previous reports implicating Adora $2 \mathrm{~b}$ in vascular functions and tissue adaptation to hypoxia (12), we next performed studies to determine the extent of tissue hypoxia in kidneys preexposed to ischemic AKI upon reopening of the renal artery. For this purpose we utilized the characteristic reduction and binding of the pimonidazole compounds to cellular macromolecules in the absence of adequate oxygen levels (12). Pimonidazole retention at baseline was only minimal, while kidney samples exposed to 30 minutes of ischemia showed robust hypoxia staining upon 10 minutes of reperfusion, indicating the persistence of renal hypoxia in spite of reestablished blood flow through the renal artery. Strikingly, the persistence of renal hypoxia in the reperfused kidney was almost completely abolished in mice pretreated with the ENT inhibitor dipyridamole (Figure 10B). In clear contrast, the effect of dipyridamole on restoring normal tissue oxygen levels during reperfusion from AKI was completely abolished in mice with endothelial deletion of Adora2b (Figure 10C). Taken together, these studies indicate the likelihood that endothelial-expressed Adora2b plays an important role in the tissue-protective effects of ENT inhibitors during ischemic AKI by helping to restore normal tissue oxygenation following ischemia.

Convergence of Ent1 and endothelial Adora $2 b$ in restoring postischemic blood flow. Prompted by the above findings of persistent tissue hypoxia during AKI reperfusion, and the remarkable interplay between ENT inhibitors and endothelial Adora2b in restoring normal tissue oxygen levels, we performed studies to address the direct effects of ENTs and ARs on renal perfusion following ischemic AKI. As first step, we used ultrasound to measure renal blood flow at baseline, during renal ischemia, and at different time points during the reperfusion period. These studies revealed that while blood flow was 
A

Adora1 ${ }^{--}$

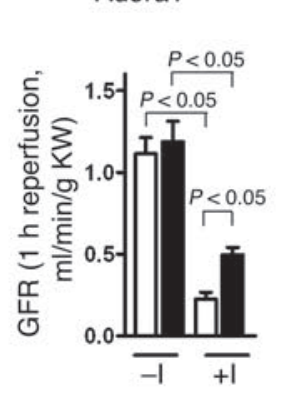

F

Adora2a $^{-1-}$

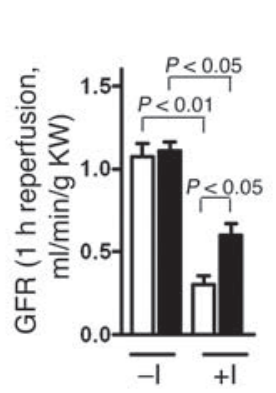

K

Adora3-

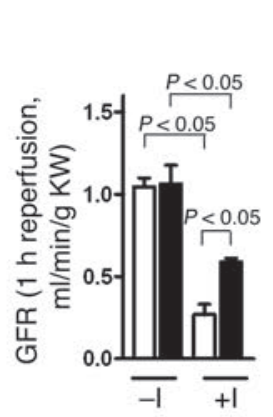

B

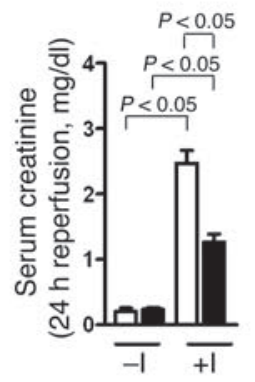

G

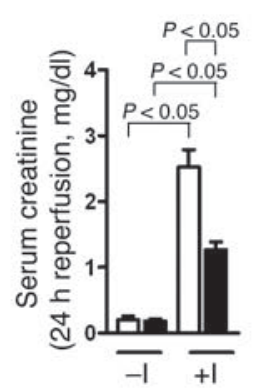

L

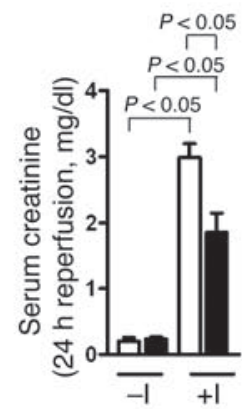

+DIP

C

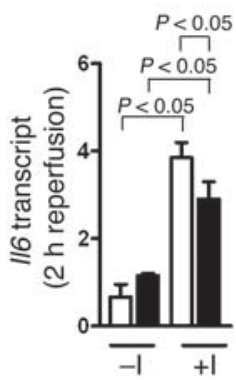

H

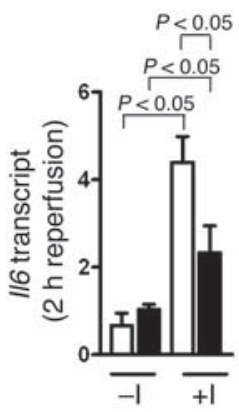

M

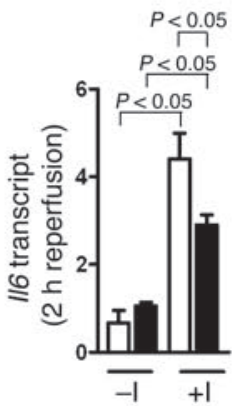

D

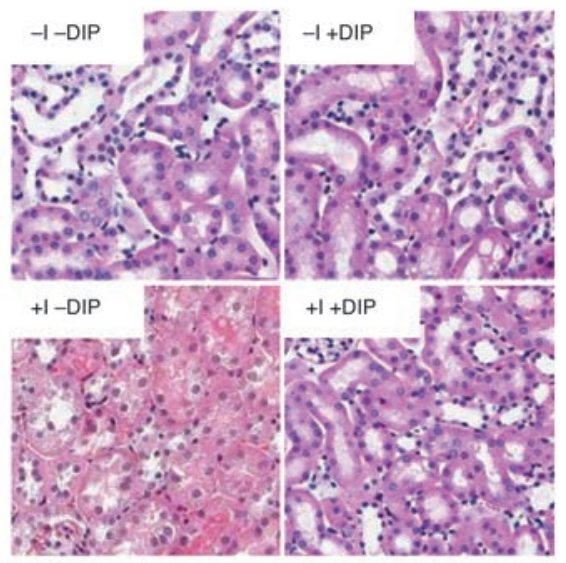

I

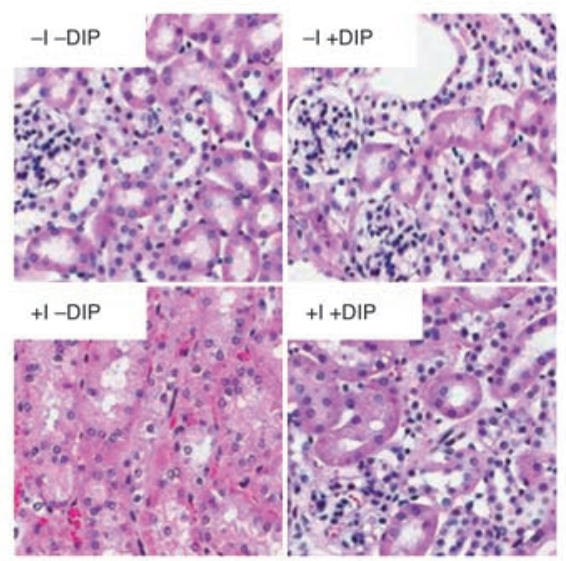

N

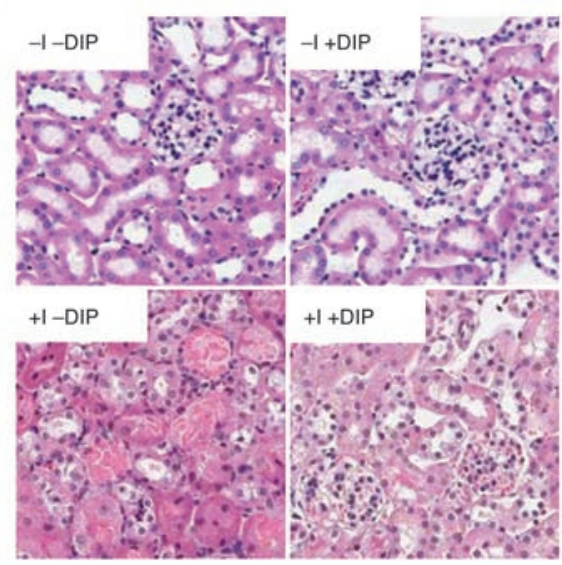

E

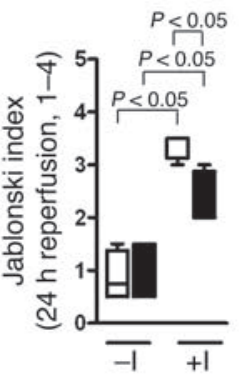

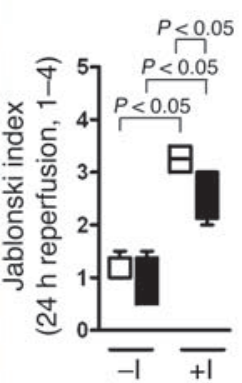

0

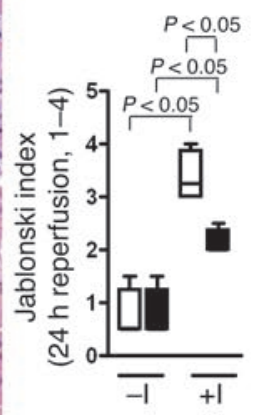

\section{Figure 7}

Treatment of ischemic AKI with dipyridamole in mice with knockout of the Adora1, Adora2a, or Adora3 AR. (A) Adora1-/- mice or littermate controls matched in sex, age, and weight and were treated with dipyridamole (+DIP: $0.25 \mathrm{mg}$ i.v.; -DIP: vehicle control) and subsequently exposed to 30 minutes of renal ischemia, and renal function was assessed by measurement of GFR following 1 hour of reperfusion $(n=4-6)$. (B) Serum creatinine levels following 30 minutes of renal ischemia and 24 hours of reperfusion $(n=4-6)$. (C) //6 transcript levels following 30 minutes of renal ischemia and 2 hours of reperfusion by real-time RT-PCR relative to $\beta$-actin $(n=4-6)$. (D) Renal histology following 30 minutes of renal ischemia and 24 hours of reperfusion (original magnification, $\times 400$; 1 representative image of 3 is shown). (E) Quantification of histologic tissue injury utilizing the Jablonski index $(n=4-6)$. $(\mathbf{F}-\mathbf{J})$ Adora2a $\mathrm{a}^{-/}$mice or littermate controls matched for sex, age, and weight $(n=4-6$ for each experimental condition). (K-O) Adora3 ${ }^{-/-}$mice or littermate controls matched for sex, age, and weight ( $n=4-6$ for each experimental condition). 


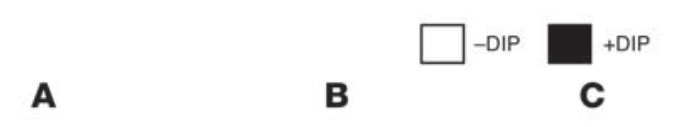

Adora2b $b^{--}$
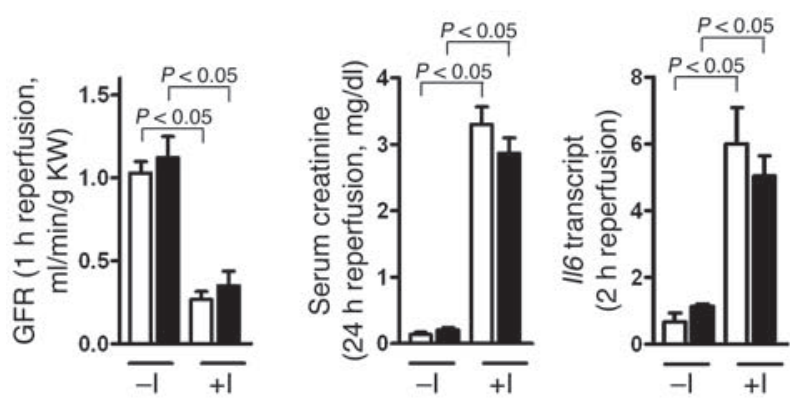

F

G

H

I

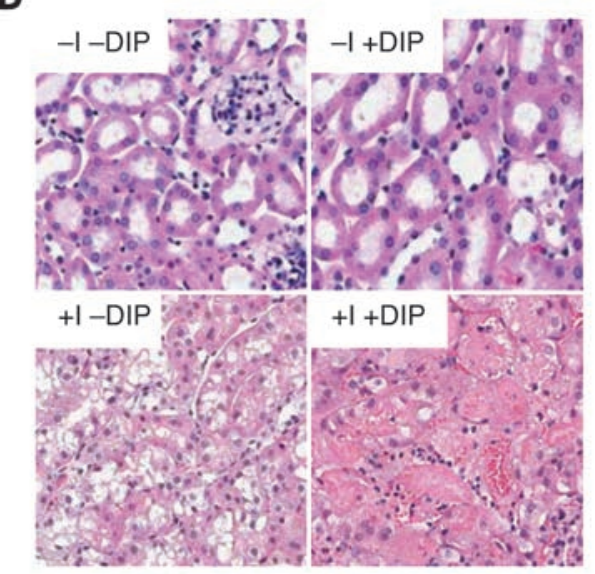

E

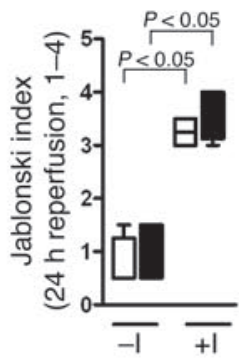

J
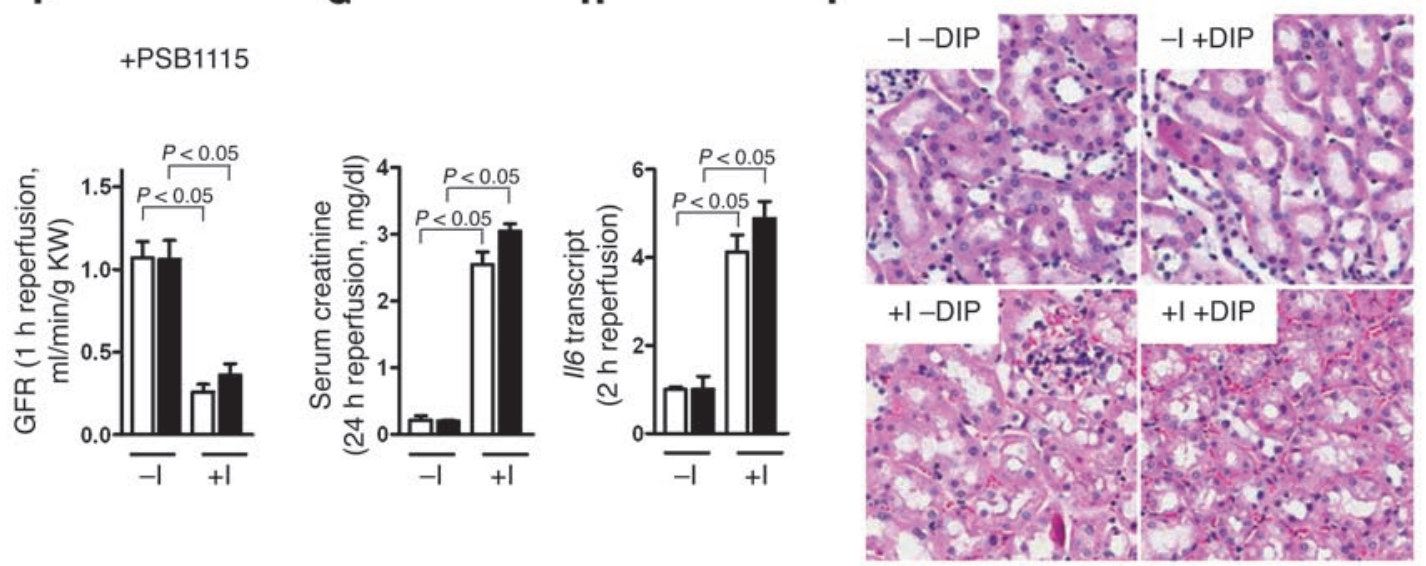

Figure 8

Role of Adora2b in mice pretreated with dipyridamole during ischemic AKI. (A) Adora2 $b^{-/-}$mice or littermate controls matched in sex, age, and weight were treated with dipyridamole (+DIP: $0.25 \mathrm{mg}$ i.v.; -DIP: vehicle control, 1 hour before renal ischemia) and subsequently exposed to 30 minutes of renal ischemia, and renal function was assessed by measurement of GFR following 1 hour of reperfusion $(n=4-6)$. (B) Serum creatinine levels following 30 minutes of renal ischemia and 24 hours of reperfusion $(n=4-6)$. (C) Measurement of //6 transcript levels following 30 minutes of renal ischemia and 2 hours of reperfusion by real-time RT-PCR relative to $\beta$-actin $(n=4)$. (D) Renal histology following 30 minutes of renal ischemia and 24 hours of reperfusion (original magnification, $\times 400$; 1 representative image of 3 is shown). (E) Quantification of histologic tissue injury utilizing the Jablonski index $(n=4)$. (A-E) Adora2 $b^{-/-}$mice or littermate controls matched in sex, age, and weight $(n=4-6$ for each experimental condition). (F-J) Wild-type mice pretreated with the Adora2b antagonist PSB1115 (0.5 mg/25 g mouse i.v.) or vehicle control ( $n=4-6$ for each experimental condition).

completely abolished during the ischemic period of renal artery ligation, cortical blood flow remained significantly diminished during the first 30 minutes of reperfusion - despite successful reopening of the renal artery (Figure 11A). Serendipitously, pretreatment with dipyridamole completely prevented this no-reflow phenomenon. In additional studies we examined cortical microvessels visualized with 10-kDa FITC-dextran and monitored via direct intravital microscopy of the externalized kidney. Indeed, postischemic microvascular flow as assessed by fluorescein intensity was dramatically diminished at 20 minutes after reopening of the renal artery (Figure 11B). Consistent with the above ultrasonographic measurements of renal perfusion, microvascular blood flow returned to baseline following pharmacologic inhibition of ENT-dependent adenosine uptake. As a next step, we examined renal perfusion by ultrasound and intravital microscopy in mice with Ent1 knockout. Consistent with our findings in dipyridamole-treated mice, the postischemic no-reflow phenomenon observed in wild-type mice was completely abolished in Ent1 $1^{-/}$mice (Figure 11, C and D). In addition, improved postischemic blood flow was entirely abolished in Ent1 $1^{-/-}$mice pretreated with PSB1115, a specific Adora2b antagonist (Figure 12, A and B). Finally, the observed increase in renal blood flow with dipyridamole treatment persisted in mice with tissue-specific deletion of Adora $2 b$ in renal epithelia. In contrast, the improved postischemic reflow with dipyridamole treatment was abolished in mice with tissue-specific deletion of Adora $2 b$ in vascular endothelia (Figure 12, C and D). In order to prove the efficiency of Ent blockade in kidney protection from ischemia, we administered dipyridamole after 30 minutes of renal ischemia. Kidney function was improved in dipyridamoletreated mice compared with mice without treatment (Figure 12E). These studies indicate that postischemic no-reflow phenomenon 
A

B

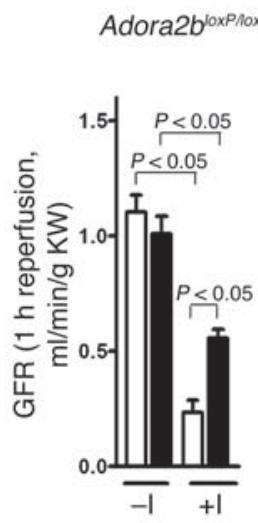

$\mathbf{F}$

G

Adora2b $b^{\text {loxploxp }}$ VE-cadherin-cre
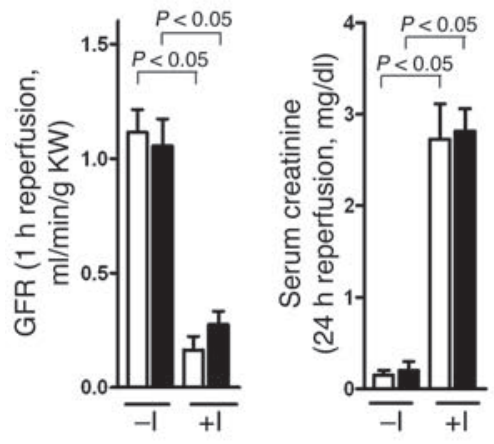

$\square$-DIP $\square$ +DIP

C

D

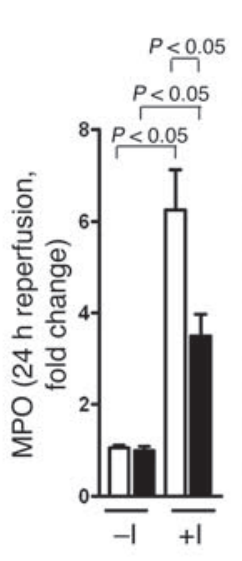

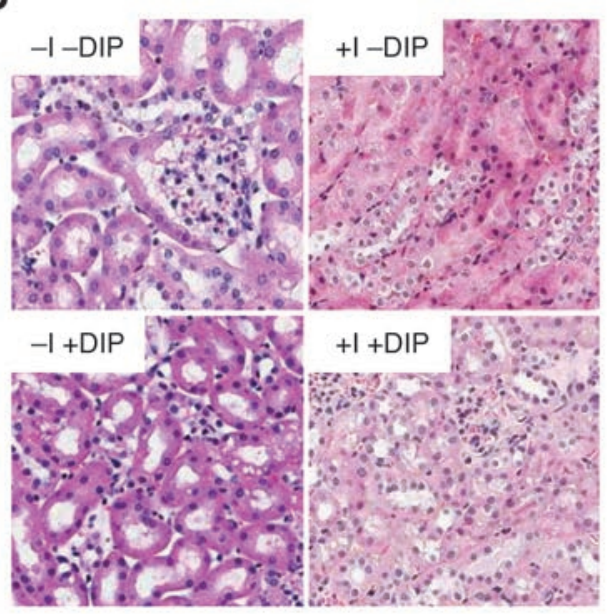

H

I

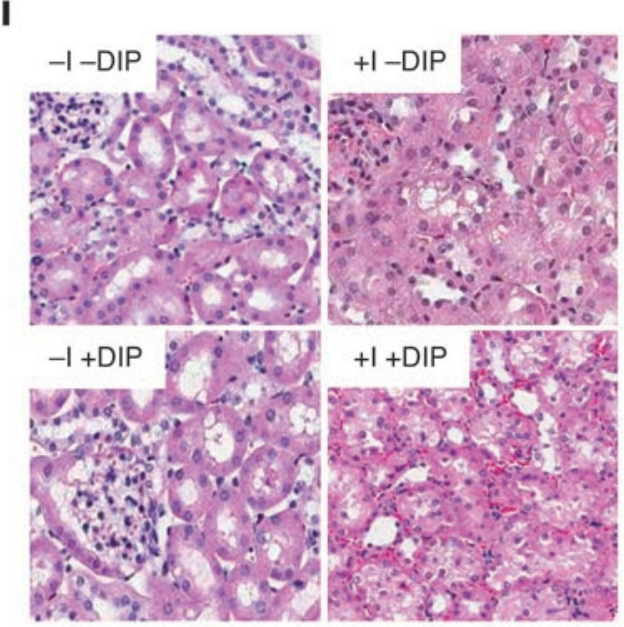

E

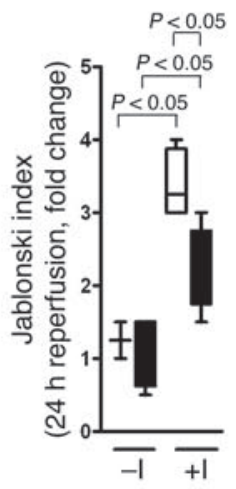

J

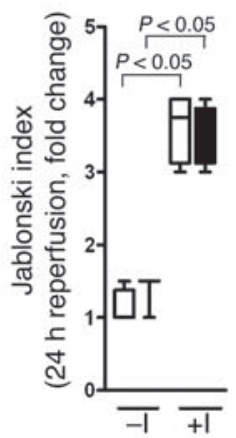

\section{Figure 9}

Consequences of tissue-specific deletion of $A d o r a 2 b$ on adenosine transport inhibitor treatment during renal ischemia. (A) Mice with deletion of

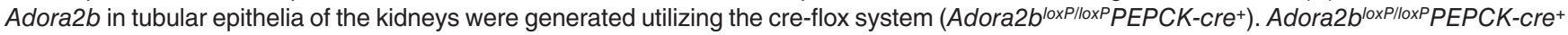
mice were treated with dipyridamole (+DIP: $0.25 \mathrm{mg}$ i.v.; -DIP: vehicle control, 1 hour before renal ischemia) and subsequently exposed to 30 minutes of renal ischemia, and renal function was assessed by measurement of GFR following 1 hour of reperfusion $(n=4)$. (B) Serum creatinine levels following 30 minutes of renal ischemia and 24 hours of reperfusion $(n=4)$. (C) Renal MPO as marker for neutrophil accumulation following 30 minutes of renal ischemia and 24 hours of reperfusion $(n=4)$. (D) Renal histology following 30 minutes of renal ischemia and 24 hours of reperfusion (original magnification, $\times 400$; 1 representative image of 3 is shown). (E) Quantification of histologic tissue injury utilizing the Jablonski index

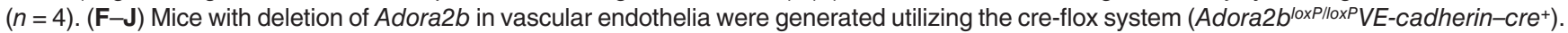
Adora2b/oxPlloxP VE-cadherin-cre ${ }^{+}$mice were treated with dipyridamole (+DIP: $0.5 \mathrm{mg}$ i.v.; -DIP: vehicle control) and subsequently exposed to 30 minutes of renal ischemia. Kidneys were studied after reperfusion for the indicated time periods $(n=4)$.

is blocked in mice with elevations in extracellular adenosine levels by pharmacologic inhibition or genetic deletion of Ent1. Moreover, our findings indicate that improved renal re-flow following renal ischemia involves signaling events through the Adora2b expressed on endothelial cells.

\section{Discussion}

Despite substantial progress in understanding the mechanisms of ischemic kidney injury (1), the therapeutic options for treating patients with AKI remain extremely limited (7). As successful reperfusion after ischemia can improve renal tissue viability and recovery, there is an urgent need to better understand and characterize molecular pathways that control renal blood flow in the post- ischemic period. Indeed, persistent microvascular dysfunction has been implicated in preventing capillary reflow despite successful opening of the arterial blood supply in several models of ischemia and reperfusion $(24,36)$, including the kidneys (37). Surprisingly, the present studies on adenosine transporters directed us toward a critical contribution of extracellular adenosine to the prevention of microvascular dysfunction following ischemic AKI. Initial studies revealed that extracellular adenosine levels can be efficiently elevated in the presence of the nucleoside transport inhibitor dipyridamole and in mice with selective deletion of the Ent1 gene. Indeed, a crosstalk pathway between renal Ent 1 and ARs of the Adora2b type expressed on vascular endothelia was critical in reestablishing renal blood flow following ischemia of the kidneys. 
A

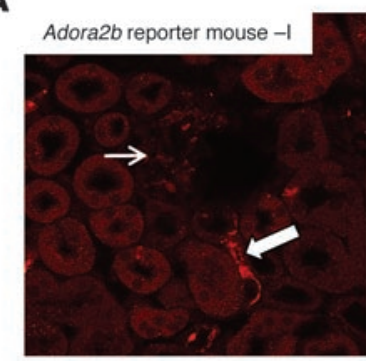

B

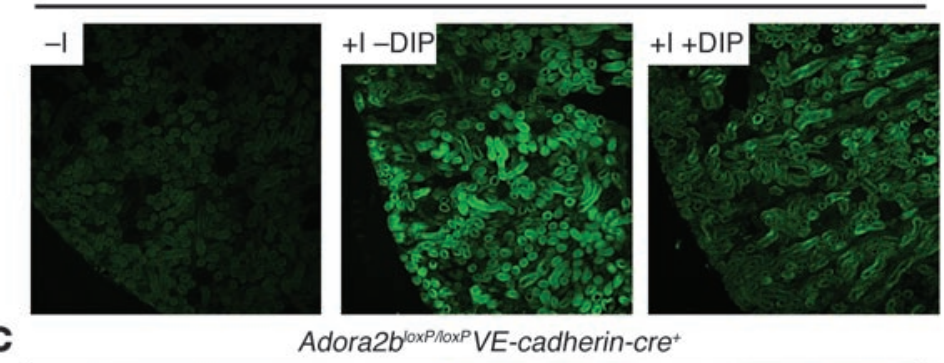

C

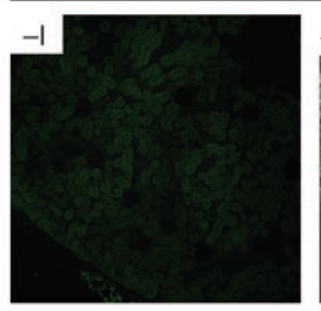

Adora2b reporter mouse +1

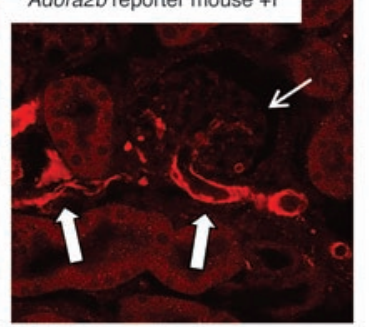

WT

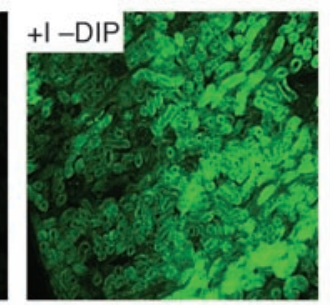

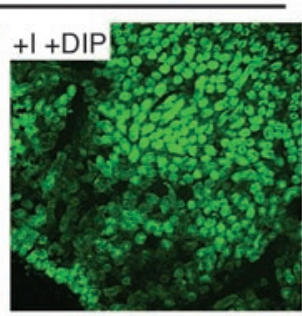

\section{Figure 10}

Renal hypoxia in mice with tissue-specific deletion of Adora2b. (A) Adora2b reporter mice (Adora2b-KO//3-galknock-in mice) were exposed to 30 minutes of renal ischemia and 24 hours of reperfusion (+I) or underwent sham operation $(-I)$. Renal tissues were stained for $\beta$-gal as an indicator of the Adora2 $b$ gene promoter, which drives expression of the reporter gene (original magnification, $\times 400$; 1 representative image of 3 is shown). Small arrows indicate the glomerulus. Bold arrows indicate Adora2b staining. (B) Staining for tissue hypoxia utilizing pimonidazole compounds that are retained in hypoxic tissues. Wild-type mice were treated with dipyridamole (0.25 mg i.v.; I+DIP) or vehicle (I-DIP; control: sham operation) and were exposed to 30 minutes of ischemia. Mice were injected with pimonidazole 5 minutes after renal ischemia, and staining was performed after 20 minutes of reperfusion (original magnification, $\times 100$; 1 representative image of 3 is shown). (C) Similar experimental setup in Adora2 $b^{\text {IoxPl/oxPVE-cadherin-Cre }}{ }^{+}$mice (1 representative image of 3 is shown).
We observed that renal ENT expression is decreased following ischemic AKI, or in renal epithelial cells exposed to ambient hypoxia. These findings are consistent with previous studies that have examined the influence of hypoxia on the expression of ENT1 or ENT2 $(22,23)$. Similar to the present results, these studies reported repression of ENT1 or ENT2 during conditions of limited oxygen availability. Moreover, the studies utilized promoter constructs and transcription factor binding assays to examine the transcriptional pathway that is responsible for ENT repression during hypoxia. Indeed, these studies identified a transcriptionally regulated pathway involving HIF. Binding of HIF to hypoxia response elements within the ENT1 or ENT2 promoter was associated with the inhibition of transcription of ENT1/2 mRNA and subsequent repression of ENT1/2 protein levels. While in most circumstances HIF functions to induce gene expression (38), the present findings are consistent with other studies showing that HIF can function to repress gene expression (20, 39-41). Together, these findings indicate that ENT repression represents an endogenous pathway to help adapt the kidney to injurious conditions, such as ischemic AKI $(20,22,23)$. Additional transcriptionally regulated pathways that enhance extracellular adenosine signaling during hypoxic conditions involve HIF-dependent repression of adenosine kinase (AK) (39) or HIF-dependent induction of the neuronal guidance molecule netrin-1 (42). Indeed, netrin-1 has been implicated in an alternative pathway of Adora $2 \mathrm{~b}$ activation $(42,43)$ and was previously shown to protect the kidneys during ischemic AKI (44). However, as the ENT1 decrease during renal ischemia observed in our study was extremely fast, it is likely that mechanisms other than HIF-dependent ENT repression - such as a rapid degradation or a proteolytic process - were contributing.
Consistent with the present findings, previous studies have shown a protective effect of extracellular adenosine during ischemic AKI. For example, genetic deletion of key enzymes responsible for the generation of extracellular adenosine $\left(\mathrm{Cd} 73^{-/-}\right.$or $\left.\mathrm{Cd} 39^{-/-}\right)$ is associated with a more severe phenotype during ischemic AKI (45-47). Moreover, other studies addressed the functional role of individual ARs. Indeed, elegant studies from the laboratories of Okusa and Linden demonstrate that signaling events through Adora2a expressed on $\mathrm{T}$ cells contributes to kidney protection from ischemia (48-51). Other studies indicate that Adora2a signaling can preserve structure and function of podocytes in renal injury models resembling diabetic nephropathy (52). Again, other studies implicate Adora1 in kidney protection from ischemia (53-56). In fact, innovative studies from the laboratory of Lee demonstrate that kidney-specific reconstitution of Adora1 via a lentiviral approach in Adora $1^{-/-}$mice is associated with reduced renal ischemia-reperfusion injury (31). Thus, the present findings demonstrating that dipyridamole-mediated kidney protection is predominantly mediated by Adora $2 b$ expressed on vascular endothelia are somewhat surprising. This could - at least in partbe related to the fact that the Adora2b is the most insensitive AR, requiring adenosine concentrations in the micromolar range $(57,58)$, whereas activation of Adora1a or Adora2a occurs at much lower adenosine concentrations. It is conceivable that particularly experimental conditions such as Ent 1 inhibition or deletion during AKI are associated with elevations in extracellular adenosine sufficient to activate Adora2b. However, we point out that even though the current studies would seem to imply that the effects of adenosine modulation in the context of ENT inhibition occur predominantly 

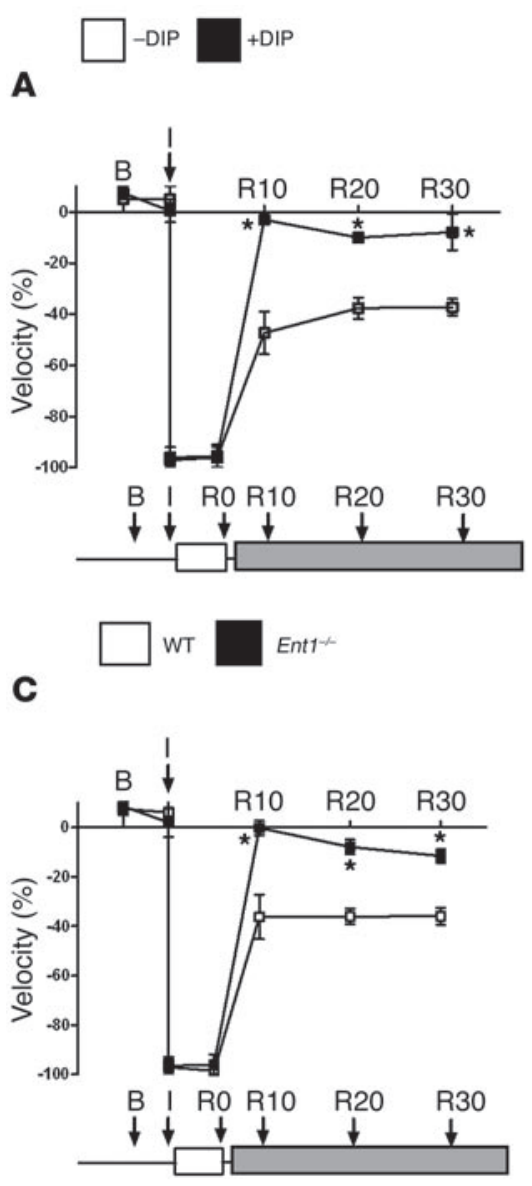

B

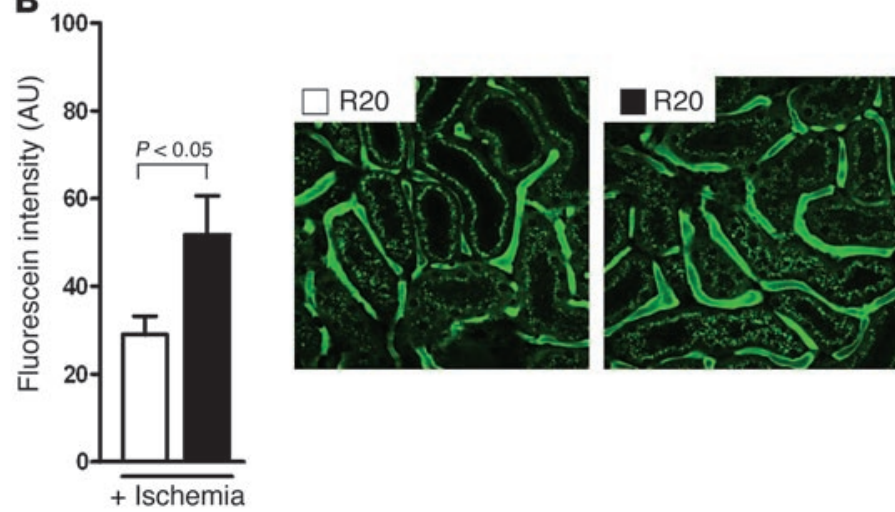

D
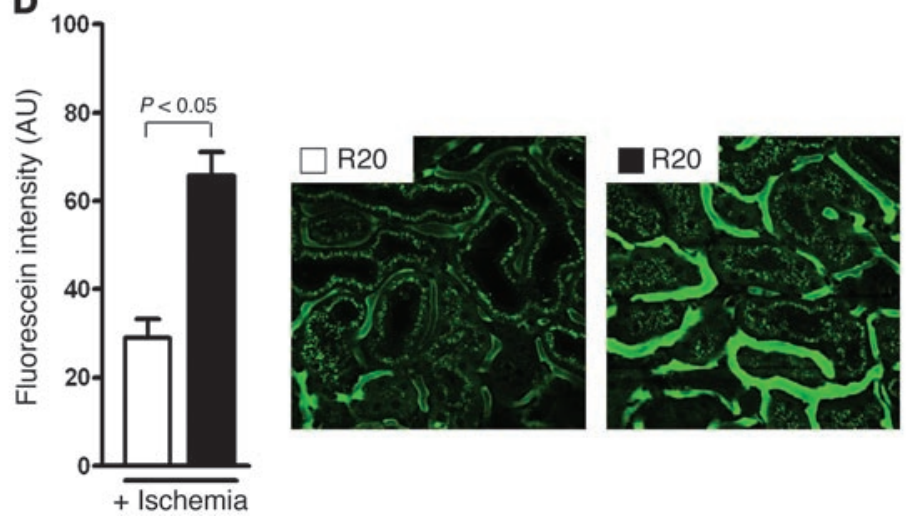

Figure 11

Role of Ent1 in regulating postischemic blood flow of the kidneys. (A) To address the consequences of dipyridamole on kidney perfusion during renal ischemia and reperfusion, we pretreated mice with dipyridamole (+DIP; $0.25 \mathrm{mg} / 25 \mathrm{~g}$ mouse i.v., 1 hour before renal ischemia) or vehicle (-DIP) and measured cortical blood flow at the indicated time points (B, baseline blood flow; I, beginning of 30 minutes of ischemia; R0-R30, reperfusion time in minutes) utilizing Doppler measurements of blood flow velocity by renal ultrasonography (B). Measurement of renal blood flow by 2-photon in vivo imaging of renal perfusion following injection of 10-kDa FITC-dextran after 30 minutes of renal ischemia and 20 minutes of reperfusion (quantification by measurements of fluorescein intensity; original magnification, $\times 400 ; n=3-6$; 1 representative image of 3 of cortical perfusion is shown). ${ }^{\star} P<0.01$ by unpaired $t$ test compared with cortical blood flow in mice treated without dipyridamole. (C and $\mathbf{D}$ ) Renal ultrasonography and 2-photon in vivo imaging in Ent1-- mice or littermate controls matched for sex, age, and weight ( $n=3-6$ in all experimental groups; original magnification, $\times 400$; 1 representative image of 3 of cortical perfusion is shown. ${ }^{\star} P<0.01$ by unpaired $t$ test, cortical blood flow in Ent1-/- mice compared with littermate controls.

via effects through Adora $2 \mathrm{~b}$ - given the apparent lack of benefit of dipyridamole in Adora $2 b^{-1-}$ mice and the complete antagonism of dipyridamole by PSB115 - it is likely that other signaling effects of adenosine may also contribute to the attenuation of AKI. Particularly, signaling effects through Adora1 or Adora2a are likely to contribute to kidney protection from AKI but may involve other tissue-specific origins (tubular epithelia, or inflammatory cells), or may contribute at a different time point of disease progression (e.g., resolution phase) (45).

Previous studies had implicated extracellular adenosine in the regulation of renal perfusion, particularly through Adora1 or Adora2a. Elegant studies from the laboratory of Schnermann provide convincing evidence that activation of Adora1 in afferent glomerular arterioles mediates the tubuloglomerular feedback mechanism - a renal vasoconstriction elicited by elevations in $\mathrm{NaCl}$ in the macula densa region of the nephron $(59,60)$. Extensions of these findings demonstrate that global elevation of renal adenosine causes steady-state vasorelaxation resulting from Adora2a-mediated generation of nitric oxide. In contrast, selective augmentation of adenosine around afferent arterioles causes persistent vasoconstriction, involving Adora1a. Thus, adenosine is a renal constrictor only when it can interact with afferent arteriolar Adora1a without affecting the bulk of renal Adora2a at the same time (61). Other studies have shown that ENT inhibition with dipyridamole is associated with an initial constriction of the afferent and efferent arterioles at an early phase and subsequent dilation at a later phase, with the same degree of vasoconstrictive and vasodilatory effect on both arterioles in rats (62). In contrast to these studies, previous studies report vasoconstrictive properties of dipyridamole during the initiation phase of postischemic acute renal failure in rats (63) or in patients with liver cirrhosis (64). Thus, we were somewhat surprised that the present findings implicate a biochemical crosstalk 


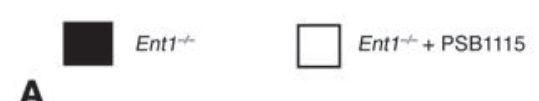

A
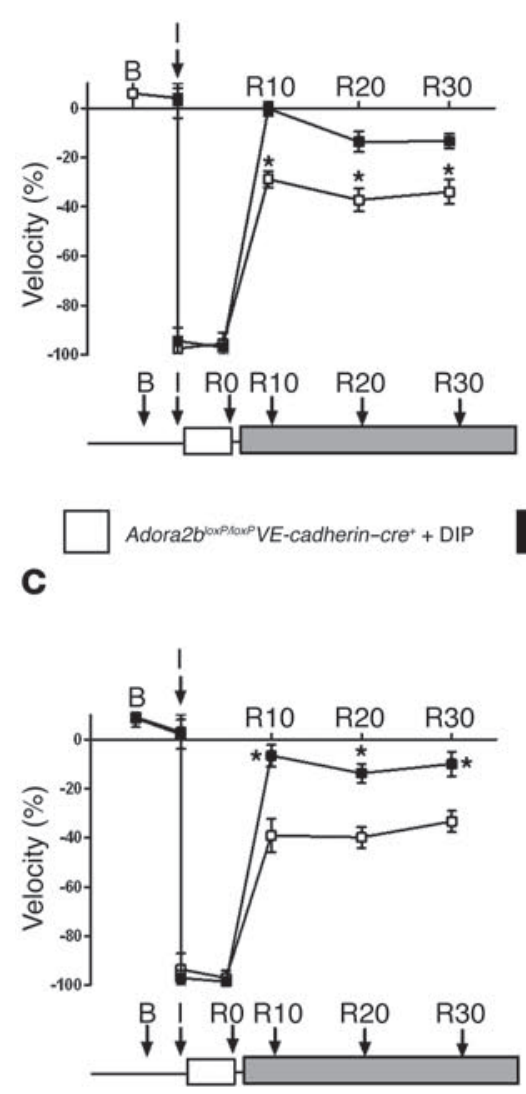

B

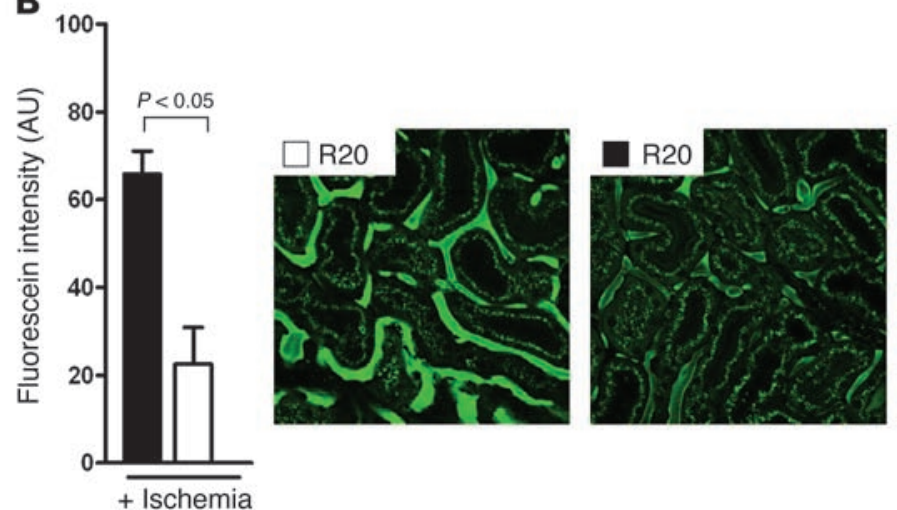

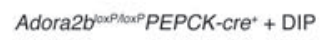

D

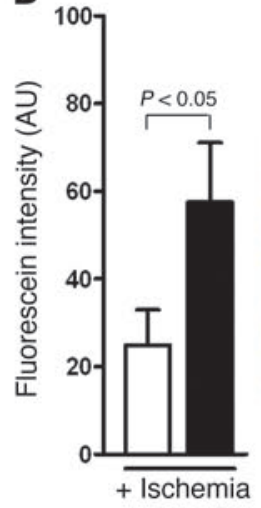

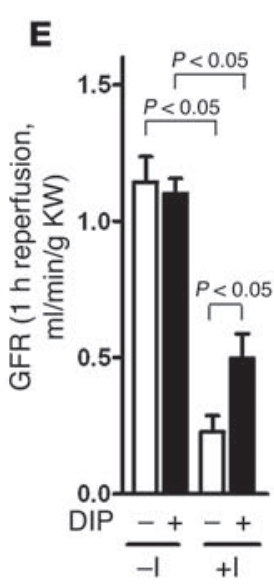

Figure 12

Interplay between Ent1 and Adora2b in regulating postischemic blood flow of the kidneys. (A and B) To address the interplay between Ent1 and Adora2b on kidney perfusion during renal ischemia and reperfusion, we pretreated Ent1 $1^{-/}$mice with PSB1115 (+PSB1115, $0.5 \mathrm{mg} / 25 \mathrm{~g}$ mouse i.v., 1 hour before renal ischemia) or vehicle (-PSB1115) and measured cortical blood flow at the indicated time points (B, baseline blood flow; I: beginning of 30 minutes ischemia; R0-R30, reperfusion time in minutes) utilizing Doppler measurements of blood flow velocity by renal ultrasonography. Renal ultrasonography and 2-photon in vivo imaging in Ent1-- mice treated with PSB1115 or vehicle control $(n=3-6$ in all experimental groups; original magnification, $\times 400$; 1 representative image of 3 ). ${ }^{*} P<0.01$ by unpaired $t$ test in Ent1 ${ }^{-/-}$mice treated with or without PSB1115. (C and D) Renal ultrasonography and 2-photon in vivo imaging in Adora2 $b^{\text {loxPlloxPPEPCK-cre }}{ }^{+}$or Adora2 $b^{\text {loxPlloxPVE-cadherin-Cre }}{ }^{+}$ mice. $n=3-6$ in all experimental groups; original magnification, $\times 400$; 1 representative image of 3 is shown. ${ }^{*} P<0.01$ by unpaired $t$ test, cortical blood flow in Adora2b $b^{\text {loxPlloxPPEPCK-cre }}{ }^{+}$compared with Adora2bloxP/loxPVE-cadherin-cre $)$. (E) To determine the time point of renal protection via Ent1, we measured GFR following 30 minutes of renal ischemia and 1 hour of reperfusion after treatment with dipyridamole (+DIP, $0.25 \mathrm{mg} / 25 \mathrm{~g}$ mouse i.v.) or vehicle (-DIP) just before reperfusion $(n=4-6)$.

pathway between ENT1 and endothelial Adora $2 \mathrm{~b}$ in the prevention of renal no-reflow following ischemic AKI. At present, it remains somewhat unclear what accounts for the opposite behavior, but this could be attributable to differences in the model systems, differences in the ENT inhibitor dose or timing, or differences in species examined. Moreover, the present studies point to the possibility that extracellular elevations in adenosine during ischemic AKI in the presence of an ENT inhibitor may become sufficient to elevate Adora2b, which is more adenosine insensitive than Adora1a or Adora2a, thereby causing vasodilation predominantly in the postischemic period (65). While the no-reflow phenomenon of the kidneys has been known for several decades $(24,37)$, there are currently no treatment modalities available that specifically prevent renal no-reflow in patients experiencing AKI. In contrast, the clinical treatment of the no-reflow phenomenon in patients undergoing a percutaneous coronary intervention (PCI) for the treatment of acute myocardial infarction are consistent with our experimental findings in murine models of ischemic AKI. Indeed, it has become routine practice to treat the no-reflow phenomenon in patients undergoing percutaneous coronary intervention (PCI) for acute myocardial infarction by direct injection of adenosine or dipyridamole into the coronary circulation $(66,67)$. Due to the fact that ischemic AKI is in most cases treated medically without vascular intervention, direct injection of adenosine or dipyridamole into the renal artery following ischemic AKI is usually not a treatment option. However, the present studies indicate several potential lines of treatment for the prevention of the no-reflow phenomenon following ischemic AKI. A first option could be the therapeutic use of 
an HIF activator that would result in repression of renal adenosine transporters, and thereby result in elevation of extracellular adenosine levels and prevention of ischemic AKI. Indeed, experimental studies implicate pharmacologic HIF activators in the treatment of renal ischemia (68), and clinical studies have shown that such compounds can be safely given for patient treatment (69). Second, patients undergoing medical procedures associated with a high risk for ischemic AKI could be prophylactically treated with the ENT inhibitor dipyridamole, which has an established safety record in patients (70). Finally, pharmacologic activators of Adora $2 \mathrm{~b}$ could be used to improve renal blood flow following ischemic AKI. Indeed, experimental studies consistent with our findings have implicated Adora2b in the dilatation of the glomerular vasculature (71). Recently, a selective agonist of Adora2b has become available (BAY 60-6583), and experimental studies have shown treatment effects of BAY 60-6583 during ischemia of the myocardium (72), intestine (19), or the kidneys (73).

Taken together, the present studies demonstrate that attenuation of renal adenosine transport through Ent1, in conjunction with signaling events involving endothelial Adora2b, contribute to the prevention of the renal no-reflow phenomenon in the setting of ischemic AKI. While these studies point toward several potential treatment options for the prevention or treatment of ischemic AKI, the present findings require translation from mice to humans. For example, the occurrence of a renal no-reflow phenomenon in patients experiencing ischemic AKI is largely unknown, as are the risk factors for developing no-reflow following renal ischemia. Moreover, the safety and efficiency of experimental therapeutics such as Adora2b agonists have to be established in patients.

\section{Methods}

Mice generation and breeding. All mice were housed in a 12-hour light/ 12-hour dark cycle and sex-, age-, and weight-matched mice were used between 12 and 16 weeks. In transcript and pharmacological studies, C57BL/6J mice obtained from The Jackson Laboratory were used. Mice deficient in Adora1, Adora2a, Adora2b, Adora3 (Adora1 ${ }^{-1-}$ mice were a gift from J. Schnermann, NIDDK, NIH, Bethesda, Maryland, USA; Adora2a-/mice were provided by Joel Linden, La Jolla Institute for Allergy and Immunology, La Jolla, California, USA; the Adora3 ${ }^{-/-}$mice were provided by Marlene Jacobson, Merck Research Laboratories, West Point, Pennsylvania, USA) or Ent 1 on the C57BL/6J strain were generated, validated, and characterized as previously described (59, 73-77). Ent2-deficient mice were obtained from Taconic. Conditional Adora2 $b^{\operatorname{lox} P / \text { loxP PEPCK-cre }{ }^{+} \text {and }}$ Adora $2 b^{\text {loxP/loxP } V E-c a d h e r i n-c r e ~} e^{+}$mice were obtained by crossing Adora $2 b^{\text {loxP } / \text { lox } P}$ with PEPCK-cre or VE-cadherin-cre mice. VE-cadherin-cre mice were obtained from The Jackson Laboratory, and Adora $2 b^{\text {loxPloxP }}$ and PEPCK-cre mice were characterized previously by collaborators $(34,78)$. In subsets of experiments, a previously described A2BAR reporter mouse on the C57BL/ 6 strain was used (Adora2b-KO/ $\beta$-gal-knock-in mice) (35).

Murine model for renal ischemia. Mice underwent right nephrectomy followed by left renal artery ischemia (30 minutes of ischemia) using a hanging weight system, as previously described (30, 46, 47, 73). Briefly, a right nephrectomy was performed, then the left kidney was isolated and the left adrenal gland dissected away. The kidney was placed in a Lucite cup and a 7-0 nylon suture threaded under the renal artery. Weights were attached at both ends of the suture, and ischemia was performed for the indicated times. Ischemia was confirmed by color change of the kidney from red to pale white. At the end of the designated ischemic time period, the weights were re-supported, and reperfusion ensued. As an additional model for ischemic AKI, we performed bilateral renal ischemia. For this purpose, both renal pedicles were simultaneously clamped for 20 minutes by utilizing microvessel clamps. At the end of surgery, mice received $0.3 \mathrm{ml}$ normal saline and were allowed to recover for 2 hours under a heat lamp for metabolic cage investigations. Inulin clearance was performed 1 hour following reperfusion.

Assessment of renal function via metabolic cage investigation. Mice were placed in metabolic cages (Tecniplast) for urine collection 2 hours following renal ischemia for 24 hours. Thereafter, plasma and urine creatinine were measured by HPLC as described below. From these data creatinine clearance was calculated. Kidneys were harvested immediately after 24 hours of reperfusion and were stored at $-80^{\circ} \mathrm{C}$ until further analysis.

Insulin clearance. Inulin clearance was measured 2 hours after renal ischemia (30 minutes) as described previously (73). Briefly, mice were anesthetized using $50 \mathrm{mg} / \mathrm{kg}$ i.p. pentobarbital. Animals were then placed on a temperature-controlled operating table to keep rectal temperature at $37^{\circ} \mathrm{C}$. The right jugular vein was cannulated for continuous infusion. Blood samples were taken via retroorbital vein plexus puncture. A catheter was placed in the urinary bladder for timed urine collection after removal of the right kidney. After surgery, mice received a bolus of $0.45 \%$ sodium chloride solution in an amount equal to $20 \%$ body weight. Continuous infusion was maintained at a rate of $800 \mu \mathrm{l} / \mathrm{h} / 25 \mathrm{~g}$ body weight, and FITC-labeled inulin $(0.75 \mathrm{~g} / 100 \mathrm{ml}$, Sigma-Aldrich) was added to the infusion for evaluation of whole kidney GFR as described before $(79,80)$. After stabilization of the animals for 20 minutes, 20-minute timed urine collections were performed. Blood was obtained in the middle of every urine collection period for measurement of FITC-inulin. Concentration of inulin in plasma and urine was determined by measurement of wavelength using a spectrophotometer (Biotek Synergy 2), and GFR was calculated by standard formulas.

Adenosine measurement via HPLC-UV and HPLC-MS/MS. Whole kidneys from sex-, age-, and weight-matched littermates were removed and immediately snap-frozen with clamps pre-cooled to the temperature of liquid nitrogen after 30 minutes of renal ischemia without reperfusion. The kidneys were pulverized under liquid nitrogen, and the tissue protein was precipitated with $0.6 \mathrm{~N}$ ice-cold perchloric acid. Tissue adenosine levels were determined via HPLC-UV as previously described $(81,82)$. To validate the HPLC-UV results, we ran the same samples with HPLC-MS/MS (Supplemental Figure 1, A and B). For this purpose, $150 \mu \mathrm{l}$ kidney perchloric acid extracts were diluted with $675 \mu \mathrm{l}$ of an aqueous solution containing a deuterated adenosine internal standard. Twenty microliters of the extracts were used for the HPLC-MS/MS analysis. An Agilent 1100 LC (Agilent Technologies) coupled to an API4000 tandem mass spectrometer with a Turbo V ion source (AB Sciex) was used for the measurement of adenosine. A stepwise gradient of $5 \mathrm{mM}$ aqueous ammonium formate buffer and increasing methanol was applied to an Ascentis RP Amide Column ( $3 \mu \mathrm{m}, 4.5 \times 150 \mathrm{~mm}$, Supelco/Sigma-Aldrich) for the chromatographic process. The electrospray ion source was operated at $500^{\circ} \mathrm{C}$ in positive ion mode with the ESI voltage set to $5,000 \mathrm{~V}$. The ion transition of $268 / 136 \mathrm{~m} / z$ was used for monitoring adenosine, with the collision energy set to $23 \mathrm{eV}$. The ratio of the adenosine signal to the deuterated internal standard signal was used for quantitation.

PEG-ADA therapy. To determine the location of adenosine, pegylated adenosine deaminase was administered i.p. 2 hours prior to ischemia. PEG-ADA was generated by the covalent modification of purified bovine ADA with activated polyethylene glycol as described previously $(83,84)$. Mice received 5 U PEG-ADA 24 hours before renal ischemia via i.p. injection. The control group was injected with saline.

Renal histology. Kidneys were excised and harvested 24 hours following 30 minutes of renal ischemia. Renal tissues were fixed in $4.5 \%$ buffered formalin, dehydrated, and embedded in paraffin. Sections $(3 \mu \mathrm{m})$ were stained with $\mathrm{H} \& \mathrm{E}$ (73). Examination and scoring of 3 representative sections of each kidney ( $n=4-6$ for each condition) were carried out in a blinded manner. 
Granulocyte staining. For analysis of neutrophil infiltration into renal tissue, slides were heated, deparaffinized, and hydrated in water. For antigen retrieval, samples were heated in Borg Decloaker, RTU (Biocare Medical) for 30 minutes at boiling point, and cooled for 20 minutes. Samples were then rinsed, and avidin solution was placed on the slides (Vector Laboratories) for 15 minutes and then rinsed. Next, biotin solution was placed on the slides (Vector Laboratories) for 15 minutes and then rinsed. Blocking solution was then applied to slides for 60 minutes at room temperature $(10 \%$ normal rabbit serum [Vector Laboratories]) in 1:1 5\% BSA and Super Block (ScyTek). Then primary antibody to neutrophils, rat anti-Ly6B.2 (AbD Serotec), was applied for 60 minutes at room temperature at a dilution of 1:100. The diluent was a 1:1 solution of 5\% BSA and Super Block. The slides were rinsed, and then secondary antibody mixture was applied for 60 minutes at room temperature at a dilution of 1:200 (Biotinylated Rabbit Anti-Rat [Vector Laboratories] diluent: 1:1, 5\% BSA and Super Block). Slides were rinsed, incubated in R.T.U. SA-HRP (Vector Laboratories) for 30 minutes at room temperature, and then rinsed again. DAB was applied (1 drop in $2 \mathrm{ml}$ ) for 5 minutes at room temperature, rinsed in $\mathrm{DI} \mathrm{H}_{2} \mathrm{O}$, counterstained with hematoxylin, dehydrated, and mounted.

TUNEL. Apoptotic cells were identified by in situ labeling of fragmented DNA using TUNEL by using the DeadEnd Fluorometric TUNEL System from Promega. Kidneys were harvested 24 hours following 30 minutes of renal ischemia. Renal tissues were fixed in $4.5 \%$ buffered formalin, dehydrated, and embedded in paraffin. Sections $(3 \mu \mathrm{m})$ were deparrafinized, and fluorescein-12-dUTP was incorporated at 3'-OH DNA ends according to the protocol provided by the manufacturer. Slides were counterstained using Vectashield with DAPI and imaged with confocal fluorescence microscopy.

Immunohistochemistry for Ent1 and Ent2. For localization of Ent 1 and Ent2 on proximal tubule cells, kidneys were harvested from wild-type mice 2 hours following 30 minutes of ischemia or without ischemia following perfusionfixation in 4\% PFA/0.1 M phosphate buffer (PB: $25 \mathrm{mM}$ sodium phosphate dibasic anhydrous, $75 \mathrm{mM}$ sodium phosphate monobasic monohydrate; $\mathrm{pH} 7.2$ ) and postfixed in the same fixative for 3 hours, followed by cryoprotection in $20 \%$ sucrose in $0.1 \mathrm{M} \mathrm{PB} \mathrm{pH} 7.2$ overnight at $4^{\circ} \mathrm{C}$. Using a cryostat, $16-\mu \mathrm{m}$ sections were collected onto Superfrost Plus slides (Fisher Scientific). The slides underwent three 10-minute washes in $0.1 \mathrm{M}$ PBS before incubation in blocking solution ( $2 \%$ normal goat serum, $1 \%$ bovine serum albumin, $0.3 \%$ Triton in PBS) for 1 hour at room temperature. The samples were then incubated overnight at $4{ }^{\circ} \mathrm{C}$ in rabbit anti-ENT1 $(1: 100)$ (Abgent) or rabbit anti-ENT2 (1:200) (Abcam) diluted in blocking solution. Following 3 washes in PBS, samples were incubated for 2 hours at room temperature with Alexa Flour 568-goat anti-rabbit (1:400) (Invitrogen) diluted in blocking buffer. The slides were washed 3 times in PBS and were then incubated with an unlabeled goat anti-rabbit IgG Fab fragment (catalog 111-297-003, Jackson ImmunoResearch Laboratories Inc.) at $4^{\circ} \mathrm{C}$ overnight. Slides were then washed 3 times in PBS and incubated with 1:200 Napilla primary antibody (Davids Biotechnologie) at $4^{\circ} \mathrm{C}$ overnight for proximal tubule counterstaining. The slides were then washed 3 times in PBS and incubated with Alexa Flour 48-goat anti-rabbit (1:400) (Invitrogen). Slides were then washed twice in PBS, followed by a 10-minute wash in $0.1 \mathrm{M} \mathrm{PB}$ and coverslipped using VECTASHIELD (Vector Laboratories).

Analysis of $\beta$-gal expression in renal sections of Adora reporter mice. To localize the Adora in renal tissues, we analyzed $\beta$-gal expression in renal sections in Adora-KO/ $\beta$-gal-knock-in mice. Kidneys were harvested following perfusion fixation in $4 \% \mathrm{PFA} / 0.1 \mathrm{M} \mathrm{PB}$ and postfixed in the same fixative for 3 hours, followed by cryoprotection in $20 \%$ sucrose in $0.1 \mathrm{M} \mathrm{PB} \mathrm{pH} 7.2$ overnight at $4{ }^{\circ} \mathrm{C}$. Using a cryostat, $16-\mu \mathrm{m}$ sections were collected onto Superfrost Plus slides (Fisher Scientific). The slides underwent three 10-minute washes in $0.1 \mathrm{M}$ PBS before incubation in blocking solution ( $2 \%$ normal goat serum, $1 \%$ BSA, $0.3 \%$ Triton in PBS) for 1 hour at room temperature. The samples were then incubated overnight at $4{ }^{\circ} \mathrm{C}$ in chicken anti- $\beta$-gal (1:500) (Aves Labs Inc.) diluted in blocking solution. Following 3 washes in PBS, samples were incubated for 2 hours at room temperature with Alexa Fluor 568-goat anti-chicken (1:400) (Invitrogen) diluted in blocking buffer. Slides were then washed twice in PBS, followed by a 10-minute wash in $0.1 \mathrm{M} \mathrm{PB}$ and coverslipped using Vectashield (Vector Laboratories).

In vivo detection of tissue hypoxia. For hypoxia staining the left kidney was exposed to renal ischemia (30 minutes). After 5 minutes of reperfusion, hypoxyprobe-1 (pimonidazole $\mathrm{HCl}$ ) was injected i.v. via the retroorbital venous plexus $(1.5 \mathrm{mg} / 25 \mathrm{~g}$ mouse in $50 \mu \mathrm{l} \mathrm{NaCl})$. Fifteen minutes thereafter, the kidneys were snap-frozen and later sliced using a cryostat. The tissue was later stained using an FITC-mAb primary antibody (Hypoxyprobe-1 Plus Kit, Natural Pharmacia International Inc.).

Renal myeloperoxidase activity. To quantify neutrophil infiltration, we performed myeloperoxidase (MPO, Hycult Technologies) measurements as previously described (85). Briefly, renal ischemia (30 minutes) was performed, and kidneys were reperfused for 24 hours. Samples were then snap-frozen and stored at $-80^{\circ} \mathrm{C}$. The assay was conducted following the manufacturer's instructions.

Compounds. For inhibition of Ent1 and Ent2, a nonspecific ENT antagonist, dipyridamole (Baxter), was administered i.v. via the retroorbital venous plexus 30 minutes before renal ischemia ( $0.5 \mathrm{mg} / 25 \mathrm{~g}$ mouse). For Adora2b receptor blockade, we used PSB1115, a highly selective, watersoluble Adora2b antagonist (86). PSB1115 (0.5 mg/25 mg mouse) was injected i.v. via the retroorbital venous plexus.

Transcriptional analysis. Total RNA was isolated from mouse kidneys or HK-2 cells using TRIzol Reagent according to the manufacturer's instructions (Invitrogen). Therefore, frozen tissue or HK-2 cell suspensions were homogenized in TRIzol Reagent and chloroform. After spinning at $12,000 \mathrm{~g}$ for 15 minutes, the aqueous phase was removed, and the RNA was precipitated with isopropanol. RNA was pelleted, washed with ethanol, and treated with DNase, and the concentration was quantified. The PCR reactions contained $1 \mu \mathrm{M}$ sense and $1 \mu \mathrm{M}$ antisense oligonucleotidase with SYBR Green (Bio-Rad). Each target sequence was amplified using increasing numbers of cycles of $94^{\circ} \mathrm{C}$ for 1 minute, $58^{\circ} \mathrm{C}$ for 0.5 minutes, $72^{\circ} \mathrm{C}$ for 1 minute. Quantification of transcript levels was measured by real-time reverse RT-PCR (iCycler, Bio-Rad). Primer sets (sense sequences, antisense sequences) are listed in Supplemental Tables 1 and 2.

Immunoblotting experiments. Ent 1 and Ent 2 content was determined in kidneys with and without ischemia, and ENT1 and ENT2 content was measured in HK-2 cells exposed to hypoxia and under normoxic conditions. For this purpose, $\mathrm{C} 57 \mathrm{BL} / 6 \mathrm{~J}$ mice were euthanized following renal ischemia at the indicated time points of reperfusion $(30,60,120)$ and immediately frozen at $-80^{\circ} \mathrm{C}$. HK- 2 cells were exposed to hypoxia for different time periods (2-48 hours). Tissues and cell suspensions were homogenized and lysed for 10 minutes in ice-cold lysis buffer $(150 \mathrm{mM} \mathrm{NaCl}, 25 \mathrm{mM}$ Tris [pH 8.0], 5 mM EDTA, $2 \%$ Triton X-100, and 10\% mammalian tissue protease inhibitor cocktail, Sigma-Aldrich) and collected in microcentrifuge tubes. After spinning at $14,000 \mathrm{~g}$ to remove cell debris, the pellet was resuspended in reducing Laemmli sample buffer and heated to $90^{\circ} \mathrm{C}$ for 5 minutes. Samples were resolved on a $12 \%$ polyacrylamide gel and transferred to a nitrocellulose membrane. The membranes were blocked for 1 hour at room temperature in PBS supplemented with $0.2 \%$ Tween 20 (PBS-T) and 4\% BSA. The membranes were incubated in $10 \mu \mathrm{g} / \mathrm{ml}$ of the respective polyclonal goat primary antibody (Santa Cruz Biotechnology Inc.) overnight at $4^{\circ} \mathrm{C}$. The membranes were washed and incubated with $1 \mu \mathrm{g} / \mathrm{ml}$ donkey anti-goat HRP conjugated with secondary antibody (Santa Cruz Biotechnology Inc.). The wash was repeated, and proteins were detected by chemiluminescence (Applied Biosystems). To control for protein loading, blots were stripped in stripping buffer for 30 minutes and washed for 10 minutes with TBST, and 
membranes were blocked for 1 hour at room temperature in PBS-T and $4 \%$ BSA. Thereafter, the membrane was incubated with $\beta$-actin, ATPase, or Ezrin (Abcam Inc.).

Generation of Ent 1 bone marrow chimeric mice. To define the contribution of renal or hematopoietic Ent1 transporters during renal ischemia, bone marrow chimeric mice were generated in which bone marrow was ablated by radiation in WT mice (C56BL/6J), followed by reconstitution with bone marrow derived from previously characterized mice with Ent1 knockout, and vice

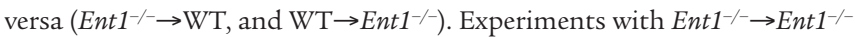
and WT $\rightarrow$ WT served as controls. In short, male donor mice (8-10 weeks old, 20-25 g) were euthanized, and the marrow from the tibia and femur was harvested by flushing the marrow cavity with sterile isotonic sodium chloride solution. The bone marrow cells were then centrifuged at $400 \mathrm{~g}$ for 5 minutes, resuspended, and counted. Recipient mice (10-12 weeks of age, $20-25 \mathrm{~g}$ ) were irradiated with a total dose of 1,100 rad (10.1 Gy) via $\mathrm{X}$-irradiation. Immediately after irradiation, $10 \times 10^{6}$ bone marrow cells/ recipient in $50 \mu \mathrm{l} 0.9 \%$ sodium chloride were injected into the retroorbital venous plexus. The resulting chimeric mice were housed in microisolators for at least 8 weeks before experimentation. Transplantation efficiency was determined by using the B6.SJL-Ptprca Pep3b/BoyJ mouse strain, with $\mathrm{CD} 45.1^{+}$bone marrow as donor bone marrow. The CD45.1 epitope, absent in cells of recipient mice, was detected by flow cytometry 8 weeks after bone marrow transplantation. The percentage of cells expressing CD45.1 was determined in each population of cells (Supplemental Figure 9).

Cell culture and treatments. HK-2 cells were obtained from ATCC and were cultured as previously described (85). Cells were exposed to hypoxia within a normobaric hypoxia chamber ( $2 \%$ oxygen, $98 \% \mathrm{~N}_{2}$ ) or normoxia for the designated times $(2,4,8,12,24$, and 48 hours). For preparation of experimental HK-2 monolayers, confluent epithelial cells were seeded at approximately $10^{5} \mathrm{cells} / \mathrm{cm}^{2}$ onto either permeable polycarbonate inserts or $75-\mathrm{cm}^{2}$ flasks.

Isolation of glomeruli and proximal tubules. The renal cortex was separated from the medulla and cut into small fragments under sterile conditions. Glomeruli were isolated by differential sieving based on the report of Harper et al. (87). The purity of glomeruli was checked by light microscopy to ensure that they were $99 \%$ free of any tubular tissue. Proximal tubules were isolated according to the method of Brezniceanu et al. $(23,88)$ by use of the Percoll gradient. Proximal tubular cells were characterized by their histological appearance as described previously (89). This procedure yielded a highly purified preparation of proximal tubules ( $97 \%$ by microscopy).

Adenosine uptake in isolated proximal tubules. Kidneys were removed from mice, and the cortex was dissected from the kidney. The cortex tissue was minced and suspended in cold DMEM-F12 with $0.1 \%$ BSA and washed 3 times. The pellet was then resuspended in $37^{\circ} \mathrm{C}$ DMEM-F12 with $0.1 \%$ BSA containing $1 \mathrm{mg} / \mathrm{ml}$ collagenase type II (Worthington Biochemical) and $25 \mathrm{mg} / \mathrm{ml}$ Trypsin Inhibitor (Invitrogen) and placed in a $37^{\circ} \mathrm{C}$ shaking incubator for 30 minutes. The solution was then poured through 40 mesh, and the cells were washed 3 times in cold DMEM-F12 with $0.1 \%$ BSA. The cells were then resuspended in cold 45\% Percoll (GE Healthcare) and 55\% $2 \times$ PBS and spun at $28,000 \mathrm{~g}$ for 15 minutes. The lower band of proximal tubule cells was removed and washed 3 times with cold PBS to remove Percoll. Cells were then resuspended in $37^{\circ} \mathrm{C}$ PBS for adenosine uptake experiments. Cells were treated with $10 \mathrm{M}$ adenosine and $10 \mathrm{M}$ dipyridamole.

Measurement of adenosine in supernatants and adenosine uptake. HK-2 cells were grown to full confluence on polycarbonate permeable supports ( $0.4 \mu \mathrm{m}$ pore, $6.5 \mathrm{~mm}$ diameter; Corning) and studied $6-8$ days after seeding. Cells were exposed as indicated to normoxia or different degrees of hypoxia. To measure adenosine uptake, $10 \mathrm{nCi}$ of ${ }^{14} \mathrm{C}$-adenosine (SigmaAldrich) was added to the supernatant on either the apical or basolateral surface of the cell monolayer. After exposure to either hypoxia or ambient air for the indicated times, samples were collected from both apical and basolateral surfaces over the indicated periods of time. Assessment of either apical or basolateral concentrations of adenosine was done by measuring the radioactivity within the lysates by directly diluting the lysate into the scintillation mixture and counting decays per minute (Liquid Scintillation Counter Beckman LS 6000IC, scintillation fluid Ecoscint A).

Measurement of renal blood flow. Kidney perfusion was assessed by 2D transabdominal ultrasound using a VisualSonics Vevo 770 high-resolution ultrasound imager equipped with a $40-\mathrm{MHz}$ transducer. Imaging was performed 10, 20, and 30 minutes after reperfusion of the ischemic left kidney (30 minutes ischemia). Supplemental anesthesia (pentobarbital) was administered as necessary. The mouse was placed in a slightly lateral supine position on an animal handling platform. Core body temperature was maintained at $37 \pm 0.5^{\circ} \mathrm{C}$ using a heated platform and a warming light, as necessary. B-mode videos and pulsed-wave Doppler tracings of the distal renal artery and cortex were routinely acquired. The angle between the mouse and the scan head was adjusted to less than $30^{\circ} \mathrm{C}$ when obtaining Doppler tracings. The built-in VisualSonics software was used to measure peak blood flow velocity and velocity-time integrals. All measurements were made in triplicate during the expiratory phase of the respiratory cycle and the values averaged for each mouse. Measurements and calculations were obtained off-line in a blinded mode.

Intravital 2-photon imaging. Imaging was performed using a confocal LSM510 Zeiss microscope equipped with an infrared femtosecond pulsed titanium-sapphire (Ti-sapphire) laser (Chameleon, Coherent Inc.) with tuning range of $690-1,020 \mathrm{~nm}$. The excitation of the 2-photon laser was set to $800 \mathrm{~nm}$ and focused onto the sample via a water immersion objective $(x 40$, 1.2 NA C-Apochromat; Zeiss). A sensitive non-descanned photo-multiplier detector collected the emission signal from the sample (dextran, 10,000 MW) after being chromatically filtered out by a band-pass HQ525/50 filter (Chroma Technology). The kidney was exposed from the mouse and dropped in a coverslip-bottomed cell culture dish in order to avoid interference by breathing motion during imaging (90). Renal ischemia was performed for 30 minutes, and dextran was injected 5 or 25 minutes after ischemia for monitoring of the perfusion recovery. During the entire observational time, the animals were kept at $37^{\circ} \mathrm{C}$. The images were analyzed for fluorescence intensity using ImageJ (http://imagej.nih.gov/ij/) imaging software. Multiple regions of interest (ROIs) were selected around the cortex. For each individual ROI, a histogram was produced and its mean value was recorded. The multiple mean intensity values corresponding to individual ROIs from the same mouse model were averaged and compared with those from different mice models. $P$ values were calculated for statistical relevance. For each mouse model $x \pm z$ ROIs out of $y$ show $P$ values less than or equal to $5 \%$, confirming that the intensity analysis is appropriate.

Biotinylation. To determine the predominant location of Ent1 and Ent2 transporters, we performed biotinylation of the apical and basolateral surfaces of HK-2 cells using Transwell Permeable Supports (Corning; pore size $0.4 \mu \mathrm{m}, 1 \times 10^{8}$ pores $/ \mathrm{cm}^{2}$ ). Cells were cultured in keratinocyte medium and grown to confluency. Tight junctions were assessed by the application of FITC-dextran, a 70-kDa protein that is approximately the size of human albumin; both FITC-dextrans and human albumin have been utilized in similar endothelial paracellular permeability models. Biotinylation was then assessed 3-5 days later according to the manufacturer's instructions (Pierce Biotechnology).

Intrarenal lentivirus delivery. Lipofectamine 2000 (Invitrogen) cotransfections were conducted with carrier and packaging vectors in HEK293 T cells as described previously (91). The carrier vector consisting of GFP-hENT1 was inserted into the plasmid pLenti6/V5-D-TOPO (Invitrogen), while the packaging vectors were PMD.G (packaging vector encoding viral envelope protein VSV-G) and pCMVdeltaR8.2 (packaging vector encoding supplementary proteins Gag, Pol, Tat, Rev; the lentiviral vectors were a gift from 
Katalin Hudak, Department of Biology, York University, Toronto, Ontario, Canada). In vivo virus transduction to express ENT1 in Ent1 $1^{-/-}$mice was performed as described by Lee et al. with slight modifications (31). In mice anesthetized with pentobarbital, the left renal pedicle was temporary occluded with a vessel clamp. Thereafter, a $31 \mathrm{G}$ needle was inserted at the lower pole of the left kidney parallel to the long axis and was pushed toward the upper pole. As the needle was slowly removed, $50 \mu$ l filter-purified lentivirus cocktail (N-GFP or N-GFP-hENT1, $10^{4} \mathrm{IU} / \mu \mathrm{l}$ ) or saline was injected. Mice were subjected to renal ischemia 24 hours after saline or virus injection. Lentivirus-mediated N-GFP or N-GFP-hENT1 protein in kidney tissue was robust after 24 hours.

Statistics. A grading scale of $0-4$, as outlined by Jablonski et al., was used for the histopathological assessment of proximal tubular damage (92). These renal injury score data are given as median and range; all other data are presented as mean \pm SD. Renal injury was analyzed with the Kruskal-Wallis test, with follow-up pairwise comparisons by Wilcoxon-MannWhitney $U$ test. Jablonski index results are shown as box-and-whisker plots. The lines within the boxes show the median; the bounds of the boxes show the upper and lower median; and the whiskers extend to the lowest and highest values. For all other outcomes, data were compared by 2-factor ANOVA with Bonferroni's post test or by 2-tailed Student's $t$ test where appropriate. Data are expressed as mean \pm SD. $P<0.05$ was considered statistically significant. For the comparison of the different ENT transcripts, pairwise comparisons were made between the control and treatment groups by unpaired 2-tailed Student's $t$ test. For Western blot analysis, 3 repeats were performed. For all statistical analysis, GraphPad Prism 5.0 software for Windows XP was used.
Study approval. All animal protocols were in accordance with the Guide for the Care and Use of Laboratory Animals (NIH publication no. 85-23. Revised 1985 ) and were approved by the Institutional Animal Care and Use Committee of the University of Colorado.

\section{Acknowledgments}

We thank Thomas Finger (Rocky Mountain Taste and Smell Center, Department of Cell and Developmental Biology, School of Medicine, University of Colorado Denver, Aurora, Colorado, USA) for support on the different immunostaining methods. We also thank the Advanced Light Microscopy Core facility, supported in part by NIH/NCRR Colorado CTSI grant UL1 RR025780. The present research work was supported by NIH grant DK081646 to V.H. Haase; a DFG (Deutsche Forschungsgemeinschaft) Research Fellowship grant (GR2121/1-1) and American Heart Association grant to A. Grenz; National Heart Institute grants R01-HL0921, R01-DK083385, R01-HL098294; and a grant from the Crohn's and Colitis Foundation of America (CCFA) to H.K. Eltzschig.

Received for publication July 28, 2011, and accepted in revised form December 7, 2011.

Address correspondence to: Holger K. Eltzschig, Mucosal Inflammation Program, Department of Anesthesiology, University of Colorado Denver, 12700 E 19th Avenue, Mailstop B112, Research Complex 2, Room 7124, Aurora, Colorado 80045, USA. Phone: 303.724.2931; Fax: 303.724.2936; E-mail: holger.eltzschig@ucdenver.edu.
1. Schrier RW, Wang W. Acute renal failure and sepsis. NEngl J Med. 2004;351(2):159-169.

2. AbueloJG. Normotensive ischemic acute renal failure. NEngl J Med. 2007;357(8):797-805.

3. Chertow GM, Burdick E, Honour M, Bonventre JV, Bates DW. Acute kidney injury, mortality, length of stay, and costs in hospitalized patients. J Am Soc Nephrol. 2005;16(11):3365-3370.

4. Gelman S. The pathophysiology of aortic crossclamping and unclamping. Anesthesiology. 1995; 82(4):1026-1060.

5. Mehta RL. Acute renal failure and cardiac surgery: marching in place or moving ahead? J Am Soc Nephrol. 2005;16(1):12-14.

6. Bove T, et al. Renoprotective action of fenoldopam in high-risk patients undergoing cardiac surgery: a prospective, double-blind, randomized clinical trial. Circulation. 2005;111(24):3230-3235.

7. Kumar AB, Suneja M. Cardiopulmonary bypassassociated acute kidney injury. Anesthesiology. 2011; 114(4):964-970.

8. Leonard MO, Cottell DC, Godson C, Brady HR, Taylor CT. The role of HIF-1 alpha in transcriptional regulation of the proximal tubular epithelial cell response to hypoxia. J Biol Chem. 2003; 278(41):40296-40304.

9. Sitkovsky MV, et al. Physiological control of immune response and inflammatory tissue damage by hypoxia-inducible factors and adenosine A2A receptors. Annu Rev Immunol. 2004;22:657-682.

10. Gladwin MT. Adenosine receptor crossroads in sickle cell disease. Nat Med. 2011;17(1):38-40.

11. Zeiser R, Penack O, Holler E, Idzko M. Danger signals activating innate immunity in graft-versushost disease. J Mol Med (Berl). 2011;89(9):833-845.

12. Eltzschig HK, Carmeliet P. Hypoxia and inflammation. N Engl J Med. 2011;364(7):656-665.

13. Fredholm BB, AP IJ, Jacobson KA, Linden J, Muller CE. International Union of Basic and Clinical Pharmacology. LXXXI. Nomenclature and classification of adenosine receptors - an update. Pharmacol Rev. 2011;63(1):1-34.
14. Fredholm BB. Adenosine, an endogenous distress signal, modulates tissue damage and repair. Cell Death Differ. 2007;14(7):1315-1323.

15. Eckle T, Kohler D, Lehmann R, El Kasmi KC, Eltzschig HK. Hypoxia-inducible factor-1 is central to cardioprotection: a new paradigm for ischemic preconditioning. Circulation. 2008;118(2):166-175.

16. Kohler D, et al. CD39/ectonucleoside triphosphate diphosphohydrolase 1 provides myocardial protection during cardiac ischemia/reperfusion injury. Circulation. 2007;116(16):1784-1794.

17. Hart ML, et al. Extracellular adenosine production by ecto-5'-nucleotidase protects during murine hepatic ischemic preconditioning. Gastroenterology. 2008; 135(5):1739-1750.

18. Hart ML, et al. Role of extracellular nucleotide phosphohydrolysis in intestinal ischemia-reperfusion injury. FASEB J. 2008;22(8):2784-2797.

19. Hart ML, Jacobi B, Schittenhelm J, Henn M, Eltzschig HK. Cutting edge: A2B adenosine receptor signaling provides potent protection during intestinal ischemia/reperfusion injury. J Immunol. 2009; 182(7):3965-3968.

20. Loffler M, Morote-Garcia JC, Eltzschig SA, Coe IR, Eltzschig HK. Physiological roles of vascular nucleoside transporters. Arterioscler Thromb Vasc Biol. 2007;27(5):1004-1013.

21. Baldwin SA, Beal PR, Yao SY, King AE, Cass CE, Young JD. The equilibrative nucleoside transporter family, SLC29. Pflugers Arch. 2004;447(5):735-743.

22. Morote-Garcia JC, Rosenberger P, Nivillac NM, Coe IR, Eltzschig HK. Hypoxia-inducible factordependent repression of equilibrative nucleoside transporter 2 attenuates mucosal inflammation during intestinal hypoxia. Gastroenterology. 2009; 136(2):607-618.

23. Eltzschig HK, et al. HIF-1-dependent repression of equilibrative nucleoside transporter (ENT) in hypoxia. J Exp Med. 2005;202(11):1493-1505.

24. Eltzschig HK, Eckle T. Ischemia and reperfusion - from mechanism to translation. Nat Med. 2011; 17(11):1391-1401.
25. Eltzschig HK, et al. Coordinated adenine nucleotide phosphohydrolysis and nucleoside signaling in posthypoxic endothelium: role of ectonucleotidases and adenosine A2B receptors. J Exp Med. 2003; 198(5):783-796.

26. Eltzschig HK, Kohler D, Eckle T, Kong T, Robson SC, Colgan SP. Central role of Sp1-regulated CD39 in hypoxia/ischemia protection. Blood. 2009; 113(1):224-232.

27. Synnestvedt K, et al. Ecto-5'-nucleotidase (CD73) regulation by hypoxia-inducible factor- 1 mediates permeability changes in intestinal epithelia. J Clin Invest. 2002;110(7):993-1002.

28. Thompson LF, et al. Crucial role for ecto-5' -nucleotidase (CD73) in vascular leakage during hypoxia. J Exp Med. 2004;200(11):1395-1405.

29. Griffiths M, et al. Cloning of a human nucleoside transporter implicated in the cellular uptake of adenosine and chemotherapeutic drugs. Nat Med. 1997; 3(1):89-93

30. Grenz A, et al. Use of a hanging-weight system for isolated renal artery occlusion during ischemic preconditioning in mice. Am J Physiol Renal Physiol. 2007; 292(1):F475-F485.

31. Kim M, Chen SW, Park SW, D’Agati VD, Yang J, Lee HT. Kidney-specific reconstitution of the A1 adenosine receptor in $\mathrm{A} 1$ adenosine receptor knockout mice reduces renal ischemia-reperfusion injury. Kidney Int. 2009;75(8):809-823.

32. Eckle T, Faigle M, Grenz A, Laucher S, Thompson LF, Eltzschig HK. A2B adenosine receptor dampens hypoxia-induced vascular leak. Blood. 2008; 111(4):2024-2035.

33. Frick JS, MacManus CF, Scully M, Glover LE, Eltzschig HK, Colgan SP. Contribution of adenosine $\mathrm{A} 2 \mathrm{~B}$ receptors to inflammatory parameters of experimental colitis. J Immunol. 2009;182(8):4957-4964.

34. Belikoff BG, et al. A2B adenosine receptor blockade enhances macrophage-mediated bacterial phagocytosis and improves polymicrobial sepsis survival in mice. J Immunol. 2011;186(4):2444-2453.

35. Yang $\mathrm{D}$, et al. The $\mathrm{A} 2 \mathrm{~B}$ adenosine receptor protects 
against inflammation and excessive vascular adhesion. J Clin Invest. 2006;116(7):1913-1923.

36. Yemisci M, Gursoy-Ozdemir Y, Vural A, Can A, Topalkara K, Dalkara T. Pericyte contraction induced by oxidative-nitrative stress impairs capillary reflow despite successful opening of an occluded cerebral artery. Nat Med.2009;15(9):1031-1037.

37. Flores J, DiBona DR, Beck CH, Leaf A. The role of cell swelling in ischemic renal damage and the protective effect of hypertonic solute. J Clin Invest. 1972; 51(1):118-126.

38. Kuhlicke J, Frick JS, Morote-Garcia JC, Rosenberger P, Eltzschig HK. Hypoxia inducible factor (HIF)-1 coordinates induction of Toll-like receptors TLR2 and TLR6 during hypoxia. PLoS One. 2007; 2(12):e1364.

39. Morote-Garcia JC, Rosenberger P, Kuhlicke J, Eltzschig HK. HIF-1-dependent repression of adenosine kinase attenuates hypoxia-induced vascular leak. Blood. 2008;111(12):5571-5580.

40. Eltzschig HK. Adenosine: an old drug newly discovered. Anesthesiology. 2009;111(4):904-915.

41. Faigle M, Seessle J, Zug S, El Kasmi KC, Eltzschig HK. ATP release from vascular endothelia occurs across $\mathrm{Cx} 43$ hemichannels and is attenuated during hypoxia. PLoS One. 2008;3(7):e2801.

42. Rosenberger P, et al. Hypoxia-inducible factordependent induction of netrin-1 dampens inflammation caused by hypoxia. Nat Immunol. 2009; 10(2):195-202.

43. Corset V, Nguyen-Ba-Charvet KT, Forcet C, Moyse E, Chedotal A, Mehlen P. Netrin-1-mediated axon outgrowth and cAMP production requires interaction with adenosine A2b receptor. Nature. 2000; 407(6805):747-750.

44. Grenz A, et al. Partial netrin-1 deficiency aggravates acute kidney injury. PLoS One. 2011;6(5):e14812.

45. Bauerle JD, Grenz A, Kim JH, Lee HT, Eltzschig HK Adenosine generation and signaling during acute kidney injury. J Am Soc Nephrol. 2011;22(1):14-20.

46. Grenz A, et al. Osswald, H. Eltzschig, HK. Protective role of ecto-5' ${ }^{\prime}$-nucleotidase (CD73) in renal ischemia. J Am Soc Nephrol. 2007;18(3):833-845

47. Grenz A, et al. Contribution of E-NTPDase 1 (CD39) to renal protection from ischemia-reperfusion injury. FASEB J. 2007;21(11):2863-2873.

48. Day YJ, et al. Renal protection from ischemia mediated by A2A adenosine receptors on bone marrowderived cells. J Clin Invest. 2003;112(6):883-891

49. Day YJ, Huang L, Ye H, Li L, Linden J, Okusa MD. Renal ischemia-reperfusion injury and adenosine $2 \mathrm{~A}$ receptor-mediated tissue protection: the role of CD4+ T cells and IFN-gamma. J Immunol. 2006; 176(5):3108-3114.

50. Okusa MD, Linden J, Macdonald T, Huang L. Selective A2A adenosine receptor activation reduces ischemia-reperfusion injury in rat kidney. $A m J$ Physiol. 1999;277(3 pt 2):F404-F412.

51. Okusa MD, Linden J, Huang L, Rieger JM, Macdonald TL, Huynh LP. A(2A) adenosine receptor-mediated inhibition of renal injury and neutrophil adhesion. Am J Physiol Renal Physiol. 2000; 279(5):F809-F818

52. Awad AS, et al. Activation of adenosine 2A receptors preserves structure and function of podocytes. J Am Soc Nephrol. 2008;19(1):59-68.

53. Lee HT, Emala CW. Protective effects of renal ischemic preconditioning and adenosine pretreatment: role of $\mathrm{A}(1)$ and $\mathrm{A}(3)$ receptors. Am J Physiol Renal Physiol. 2000;278(3):F380-F387.

54. Lee HT, Emala CW. Preconditioning and adenosine protect human proximal tubule cells in an in vitro model of ischemic injury. J Am Soc Nephrol. 2002; 13(11):2753-2761.

55. Lee HT, Gallos G, Nasr SH, Emala CW. A1 adenosine receptor activation inhibits inflammation, necrosis, and apoptosis after renal ischemiareperfusion injury in mice. J Am Soc Nephrol. 2004; 15(1):102-111.

56. Gallos G, Ruyle TD, Emala CW, Lee HT. A1 adenosine receptor knockout mice exhibit increased mortality, renal dysfunction, and hepatic injury in murine septic peritonitis. Am J Physiol Renal Physiol. 2005; 289(2):F369-F376

57. Aherne CM, Kewley EM, Eltzschig HK. The resurgence of A2B adenosine receptor signaling. Biochim Biophys Acta. 2011;1808(5):1329-1339.

58. Koeppen M, Eckle T, Eltzschig HK. Interplay of hypoxia and $\mathrm{A} 2 \mathrm{~B}$ adenosine receptors in tissue protection. Adv Pharmacol. 2011;61:145-186.

59. Sun D, et al. Mediation of tubuloglomerular feedback by adenosine: evidence from mice lacking adenosine 1 receptors. Proc Natl Acad Sci U S A. 2001 98(17):9983-9988.

60. Castrop H, et al. Impairment of tubuloglomerular feedback regulation of GFR in ecto- $5^{\prime}$-nucleotidase/CD73-deficient mice. J Clin Invest. 2004; 114(5):634-642.

61. Hansen PB, Hashimoto S, Oppermann M, Huang Y, Briggs JP, Schnermann J. Vasoconstrictor and vasodilator effects of adenosine in the mouse kidney due to preferential activation of A1 or A2 adenosine receptors. J Pharmacol Exp Ther. 2005; 315(3):1150-1157.

62. Nakamoto H, Ogasawara Y, Kajiya F. Visualisation of the effects of dilazep on rat afferent and efferent arterioles in vivo. Hypertens Res. 2008;31(2):315-324.

63. Lin JJ, Churchill PC, Bidani AK. The effect of dipyridamole on the initiation phase of postischemic acute renal failure in rats. Can J Physiol Pharmacol. 1987; 65(7):1491-1495.

64. Llach J, et al. Effect of dipyridamole on kidney function in cirrhosis. Hepatology. 1993;17(1):59-64.

65. Grenz A, Homann D, Eltzschig HK. Extracellular adenosine: a safety signal that dampens hypoxiainduced inflammation during ischemia. Antioxid Redox Signal. 2011;15(8):2221-2234.

66. Tanzilli G, et al. Dipyridamole versus verapamil for treatment of no-reflow during primary angioplasty. Catheter Cardiovasc Interv. 2010;76(6):787-793.

67. Marzilli M, Orsini E, Marraccini P, Testa R. Beneficial effects of intracoronary adenosine as an adjunct to primary angioplasty in acute myocardial infarction. Circulation. 2000;101(18):2154-2159.

68. Hill $\mathrm{P}$, et al. Inhibition of hypoxia inducible factor hydroxylases protects against renal ischemia-reperfusion injury. J Am Soc Nephrol. 2008;19(1):39-46.

69 . Bernhardt WM, et al. Inhibition of prolyl hydroxylases increases erythropoietin production in ESRD. J Am Soc Nephrol. 2010;21(12):2151-2156.

70. Sacco RL, et al. Aspirin and extended-release dipyridamole versus clopidogrel for recurrent stroke. N Engl J Med. 2008;359(12):1238-1251.

71. Al-Mashhadi RH, Skott O, Vanhoutte PM, Hansen $\mathrm{PB}$. Activation of $\mathrm{A}(2)$ adenosine receptors dilates cortical efferent arterioles in mouse. Kidney Int. 2009; 75(8):793-799.

72. Eckle T, et al. Cardioprotection by ecto- 5 '-nucleotidase (CD73) and A2B adenosine receptors. Circulation. 2007;115(12):1581-1590.

73. Grenz A, et al. The reno-vascular A2B adenosine receptor protects the kidney from ischemia. PLoS
Med. 2008;5(6):e137.

74. Yang $Z$, et al. Infarct-sparing effect of A2A-adenosine receptor activation is due primarily to its action on lymphocytes. Circulation. 2005;111(17):2190-2197.

75. Salvatore CA, Tilley SL, Latour AM, Fletcher DS, Koller BH, Jacobson MA. Disruption of the A(3) adenosine receptor gene in mice and its effect on stimulated inflammatory cells. J Biol Chem. 2000; 275(6):4429-4434.

76. Rose JB, et al. Equilibrative nucleoside transporter 1 plays an essential role in cardioprotection. $A m J$ Physiol Heart Circ Physiol. 2010;298(3):H771-H777.

77. Choi DS, et al. The type 1 equilibrative nucleoside transporter regulates ethanol intoxication and preference. Nat Neurosci. 2004;7(8):855-861.

78. Rankin EB, Tomaszewski JE, Haase VH. Renal cyst development in mice with conditional inactivation of the von Hippel-Lindau tumor suppressor. Cancer Res. 2006;66(5):2576-2583.

79. Lorenz JN, Gruenstein E. A simple, nonradioactive method for evaluating single-nephron filtration rate using FITC-inulin. Am J Physiol. 1999; 276(1 Pt 2):F172-F177.

80. Conger JD, Falk SA, Yuan BH, Schrier RW. Atrial natriuretic peptide and dopamine in a rat model of ischemic acute renal failure. Kidney Int. 1989; 35(5):1126-1132.

81. Delabar U, Kloor D, Luippold G, Muhlbauer B. Simultaneous determination of adenosine, S-adenosylhomocysteine and S-adenosylmethionine in biological samples using solid-phase extraction and high-performance liquid chromatography. J Chromatogr B Biomed Sci Appl. 1999;724(2):231-238.

82. Kloor D, Delabar U, Muhlbauer B, Luippold G, Osswald $\mathrm{H}$. Tissue levels of S-adenosylhomocysteine in the rat kidney: effects of ischemia and homocysteine. Biochem Pharmacol. 2002;63(4):809-815.

83. Young HW, et al. A3 adenosine receptor signaling contributes to airway inflammation and mucus production in adenosine deaminase-deficient mice. J Immunol. 2004;173(2):1380-1389.

84. Zhang Y, et al. Detrimental effects of adenosine signaling in sickle cell disease. Nat Med. 2011;17(1):79-86.

85. Grenz A, et al. Partial netrin-1 deficiency aggravates acute kidney injury. PLoS One. 2011;6(5):e14812.

86. Yan L, Muller CE. Preparation, properties, reactions, and adenosine receptor affinities of sulfophenylxanthine nitrophenyl esters: toward the development of sulfonic acid prodrugs with peroral bioavailability. J Med Chem. 2004;47(4):1031-1043.

87. Harper PA, Robinson JM, Hoover RL, Wright TC, Karnovsky MJ. Improved methods for culturing rat glomerular cells. Kidney Int. 1984;26(6):875-880.

88. Brezniceanu ML, et al. Transforming growth factorbeta 1 stimulates angiotensinogen gene expression in kidney proximal tubular cells. Kidney Int. 2006; 69(11):1977-1985.

89. Vinay P, Gougoux A, Lemieux G. Isolation of a pure suspension of rat proximal tubules. Am JPhysiol. 1981; 241(4):F403-F411.

90. Dunn KW, et al. Functional studies of the kidney of living animals using multicolor two-photon microscopy. Am J Physiol Cell Physiol. 2002;283(3):C905-C916.

91. Reyes G, et al. Characterization of mammalian equilibrative nucleoside transporters (ENTs) by mass spectrometry. Protein Expr Purif. 2010; 73(1):1-9.

92. Jablonski P, Howden BO, Rae DA, Birrell CS, Marshall VC, Tange J. An experimental model for assessment of renal recovery from warm ischemia. Transplantation. 1983;35(3):198-204. 